\title{
Wiley StatsRef: Statistics Reference Online
}

\section{Mixed Outcomes}

\section{By Geert Molenberghs ${ }^{1,2}$ and Geert Verbeke $e^{2,1}$}

Keywords: mixed outcome, repeated measures, developmental toxicity, random effects, hierarchical data

\begin{abstract}
In this article, we give an overview of the joint modeling of outcomes of a mixed type, both when these outcomes are measured once each, as well as when they are measured repeatedly. Specific attention is given to the setting of surrogate marker evaluation and risk assessment in the context of developmental toxicity studies. Data analysis illustrates the methods. Brief remarks are made about the available software.
\end{abstract}

\section{Introduction}

Statistical problems where various outcomes of a different data type, that is, mixed outcomes, are observed have been around for a long time now and are rather common at present. Perhaps, the most common situation, whether in psychometry (see Psychometrics, Overview), biometry, or other fields, is that of the joint occurrence of a continuous, often normally distributed, and a binary or ordinal outcome. Emphasis can be placed on the determination of the entire joint distribution of both outcomes, or on specific aspects, such as the association in general or correlation in particular between both outcomes.

It is useful to distinguish between three approaches. We explain this in terms of a continuous and a binary outcome, but other situations can be envisaged in the same way. We return to this in what follows. The first one postulates a marginal model for the binary outcome and then formulates a conditional model for the continuous outcome, given the categorical one. For the former, one can use logistic regression, whereas for the latter conditional normal models are a straightforward choice, that is, a normal model with the categorical outcome used as a covariate ${ }^{[1]}$. The second family starts from the reverse factorization, combining a marginal model for the continuous outcome with a conditional one for the categorical outcome. Conditional models have been discussed by Cox and Wermuth ${ }^{[2,3]}$, Krzanowski ${ }^{[4]}$, and Little and Schluchter ${ }^{[5]}$. Schafer ${ }^{[6]}$ presents a so-called general location model where a number of continuous and binary outcomes can be modeled together.

1 Universiteit Hasselt, Diepenbeek, Belgium

${ }^{2} \mathrm{KU}$ Leuven, Leuven, Belgium

Update based on original article by Geert Molenberghs, Wiley StatsRef: Statistics Reference Online $\odot 2014$ John Wiley \& Sons, Ltd.

Wiley StatsRef: Statistics Reference Online, @ 2014-2018 John Wiley \& Sons, Ltd.

This article is $\odot 2018$ John Wiley \& Sons, Ltd.

DOI: 10.1002/9781118445112.stat07623.pub2 
The third model family directly formulates a joint model for the two outcomes. In this context, one often starts from a bivariate continuous variable, one component of which is explicitly observed and the other one observed in dichotomized, or generally discretized, version only ${ }^{[7]}$. Molenberghs et al. ${ }^{[8]}$ presented a model based on a Plackett-Dale approach, where a bivariate Plackett distribution is assumed, of which one margin is directly observed and the other one only after dichotomization. General multivariate exponential family-based models have been proposed by Prentice and Zhao ${ }^{[9]}$, Zhao et al. ${ }^{[10]}$, and Sammel et al. ${ }^{[11]}$.

Of course, these developments have not been limited to bivariate joint outcomes. One can obviously extend these ideas and families to a multivariate continuous outcome and/or a multivariate categorical outcome. For the first and second families, one then starts from conditional and marginal multivariate normal and appropriately chosen multinomial models. Such a model within the first family has been formulated by Olkin and Tate ${ }^{[12]}$. Within the third family, early models were formulated by Hannan and Tate ${ }^{[13]}$ and $\mathrm{Cox}^{[14]}$ for a multivariate normal with a univariate, bivariate, or discrete variable.

Apart from an extension from the bivariate to the multivariate case, one can introduce other hierarchies as well. For example, each of the outcomes may be measured repeatedly (see Repeated Measures) over time, and there could even be several repeated outcomes in both the continuous and the categorical subgroup. Terminology frequently used in this context is joint models, multivariate hierarchical, and multivariate longitudinal data (see Longitudinal Data Analysis). We return to this case in Section 3.

A very specific hierarchy stems from clustered data, where a continuous and a categorical subgroup, or several of each, are observed for each member of a family, a household, a cluster, and so on. For the specific context of developmental toxicity studies, often conducted in rats and mice, a number of developments have been made. An overview of such methods, together with developments for probit-normal and Plackett-Dale-based models, was presented in Regan and Catalano ${ }^{[15]}$. Catalano and Ryan ${ }^{[16]}$ and Fitzmaurice and Laird ${ }^{[17]}$ propose models for a combined continuous and discrete outcome, but differ in the choice of which outcome to condition on the other one. Both use generalized estimating equations (GEE) to allow for clustering. Catalano ${ }^{[18]}$ extended the model by Catalano and Ryan ${ }^{[16]}$ to accommodate ordinal variables.

Regan and Catalano ${ }^{[19]}$ proposed a probit-type model to accommodate joint continuous and binary outcomes in a clustered data context, thus extending the correlated probit model for binary outcomes ${ }^{[20]}$ to incorporate continuous outcomes. Geys et al. ${ }^{[21]}$ used a Plackett latent variable to the same effect, extending the bivariate version proposed by Molenberghs et al. ${ }^{[8]}$. Estimation in such hierarchical joint models can be challenging. Regan and Catalano ${ }^{[19]}$ proposed maximum likelihood, but considered GEE $^{[22]}$ as an option too ${ }^{[23]}$. Geys et al. ${ }^{[21]}$ made use of pseudo-likelihood. Ordinal extensions have been proposed in Regan and Catalano ${ }^{[24]}$. We return to the developmental toxicity case in Section 5.

It is clear that the literature on joint modeling of outcomes of various natures is diverse and growing. A broad ranging review of hierarchical models for joint continuous and discrete models can be found in Regan and Catalano ${ }^{[15]}$. Here, we will focus on a few selected but generic methods. We will emphasize the case of a continuous and a binary outcome as a basic paradigm (Section 2). In particular, a probit-normal formulation will be developed (Section 2.1), a Plackett-Dale approach (Section 2.2), and a bivariate generalized linear mixed model (GLMM) of a joint nature (Section 2.3). Hierarchical versions will be discussed in Section 3. In Section 4, using data from an ophthalmology study, used in the context of surrogate endpoint evaluation, the methods presented will be illustrated. In Section 5 , the specific case of risk assessment is discussed. Section 6 discusses high-dimensional settings. Brief software considerations are offered in Section 7. The developments in this overview are largely based on Molenberghs and Verbeke [25, Ch. 24 and 25].

Wiley StatsRef: Statistics Reference Online, (c) 2014-2018 John Wiley \& Sons, Ltd.

This article is $\odot 2018$ John Wiley \& Sons, Ltd.

DOI: 10.1002/9781118445112.stat07623.pub2 


\section{A Continuous and a Binary Endpoint}

In this section, we start off with the bivariate, nonhierarchical, setting. Extensions to the fully hierarchical case are the topic of Section 3.

Two modeling strategies can be considered to accommodate mixed binary-continuous endpoints, because the joint distribution of a mixed continuous-discrete outcome vector can always be expressed as the product of the marginal distribution of one of the responses and the conditional distribution of the remaining response given the former response. One can choose either the continuous or the discrete outcome for the marginal model. The main problem with such approaches is that no easy expressions for the association between both endpoints are obtained. Therefore, we opt for a more symmetric treatment of the two outcome variables.

Let the two outcomes for subject $i(i=1, \ldots, N)$ be $S_{i}$ and $T_{i}$. The connotation is that $S$ is a surrogate endpoint for $T$, but this particular setting implies no limitation on the use of the framework whatsoever.

We treat the case where the surrogate is binary and the true endpoint is continuous. The reverse case is entirely similar. Details on the surrogacy setting can be found in Burzykowski et al. ${ }^{[26]}$ and Alonso et al. ${ }^{[27]}$.

Let $\tilde{S}_{i}$ be a latent variable of which $S_{i}$ is the dichotomized version. In Section 2.1, we will describe a bivariate normal model for $\tilde{S}_{i}$ and $T_{i}$, resulting in a probit-linear model for $S_{i}$ and $T_{i}$. Section 2.2 presents an alternative formulation based on the bivariate ${ }^{[28]}$ density, resulting in a Plackett-Dale model.

\subsection{A Probit-Normal Formulation}

In this formulation, we assume the following model:

$$
\begin{aligned}
& T_{i}=\mu_{T}+\beta X_{i}+\varepsilon_{T i} \\
& \tilde{S}_{i}=\mu_{S}+\alpha X_{i}+\varepsilon_{S i}
\end{aligned}
$$

where $\mu_{S}$ and $\mu_{T}$ are fixed intercepts and $\alpha$ and $\beta$ are the fixed effects of the treatment $X$ on the surrogate and true endpoints, respectively. Further, $\varepsilon_{S i}$ and $\varepsilon_{T i}$ are correlated error terms, assumed to satisfy:

$$
\left(\begin{array}{c}
\varepsilon_{T i} \\
\varepsilon_{S i}
\end{array}\right) \sim N\left[\left(\begin{array}{l}
0 \\
0
\end{array}\right),\left(\begin{array}{cc}
\sigma^{2} & \frac{\rho \sigma}{\sqrt{1-\rho^{2}}} \\
& \frac{1}{1-\rho^{2}}
\end{array}\right)\right]
$$

Models (1) and (2) specify a bivariate normal density. The variance of $\tilde{S}_{i}$ is chosen for reasons that will be made clear in what follows. From these models, it is easily seen that the density of $T_{i}$ is univariate normal with regression given in Equation (1) and variance $\sigma^{2}$, implying that the parameters $\mu_{T}$, $\beta$, and $\sigma^{2}$ can be estimated using linear regression software with response $T_{i}$ and single covariate $Z_{i}$. Similarly, the conditional density of $\tilde{S}_{i}$, given $X_{i}$ and $T_{i}$, is

$$
\tilde{S}_{i} \sim N\left[\left(\mu_{S}-\frac{\rho}{\sigma \sqrt{1-\rho^{2}}} \mu_{T}\right)+\left(\alpha-\frac{\rho}{\sigma \sqrt{1-\rho^{2}}} \beta\right) X_{i}+\frac{\rho}{\sigma \sqrt{1-\rho^{2}}} T_{i} ; 1\right]
$$

having unit variance and thus motivating our earlier choice for the covariance matrix of $T_{i}$ and $\tilde{S}_{i}$. The corresponding probability is

$$
P\left(S_{i}=1 \mid T_{i}, X_{i}\right)=\Phi_{1}\left(\lambda_{0}+\lambda_{X} X_{i}+\lambda_{T} T_{i}\right)
$$

Wiley StatsRef: Statistics Reference Online, @ 2014-2018 John Wiley \& Sons, Ltd.

This article is @ 2018 John Wiley \& Sons, Ltd.

DOI: 10.1002/9781118445112.stat07623.pub2 
where

$$
\begin{aligned}
& \lambda_{0}=\mu_{S}-\frac{\rho}{\sigma \sqrt{1-\rho^{2}}} \mu_{T} \\
& \lambda_{X}=\alpha-\frac{\rho}{\sigma \sqrt{1-\rho^{2}}} \beta \\
& \lambda_{T}=\frac{\rho}{\sigma \sqrt{1-\rho^{2}}}
\end{aligned}
$$

and $\Phi_{1}$ is the standard normal cumulative density function. Note that Equation (5) implicitly defines the cutoff value for the dichotomized version. The $\lambda$ parameters can be found by fitting model (5) to $S_{i}$ with covariates $X_{i}$ and $T_{i}$. This can be done with standard logistic regression software if it allows to specify the probit rather than the logit link, such as the LOGISTIC and GENMOD procedures in SAS ${ }^{\oplus}$. Given the parameters from the linear regression on $T_{i}\left(\mu_{T}, \beta\right.$, and $\left.\sigma^{2}\right)$ and the probit regression on $S_{i}\left(\lambda_{0}, \lambda_{X}\right.$, and $\left.\lambda_{T}\right)$, the parameters from the linear regression on $\tilde{S}_{i}$ can now be obtained from Equations (6) $-(8)$ :

$$
\begin{aligned}
\mu_{S} & =\lambda_{0}+\lambda_{T} \mu_{T} \\
\alpha & =\lambda_{Z}+\lambda_{X} \beta \\
\rho^{2} & =\frac{\lambda_{T}^{2} \sigma^{2}}{1+\lambda_{T}^{2} \sigma^{2}}
\end{aligned}
$$

The asymptotic covariance matrix of the parameters $\left(\mu_{T}, \beta\right)$ can be found from standard linear regression output. The variance of $\hat{\sigma}^{2}$ equals $2 \sigma^{4} / N$. The asymptotic covariance of $\left(\hat{\lambda}_{0}, \hat{\lambda}_{X}, \hat{\lambda}_{T}\right)$ follows from logistic (probit) regression output. These three statements yield the covariance matrix of the six parameters upon noting that it is block diagonal. To derive the asymptotic covariance of $\left(\mu_{S}, \alpha, \rho\right)$, it suffices to calculate the derivatives of Equations (9) - (11) with respect to the six original parameters and apply the delta method. They are:

$$
\frac{\partial\left(\mu_{S}, \alpha, \rho\right)}{\partial\left(\mu_{T}, \beta, \sigma^{2}, \lambda_{0}, \lambda_{X}, \lambda_{T}\right)}=\left(\begin{array}{cccccc}
\lambda_{T} & 0 & 0 & 1 & 0 & \mu_{T} \\
0 & \lambda_{T} & 0 & 0 & 1 & \beta \\
0 & 0 & h_{1} & 0 & 0 & h_{2}
\end{array}\right)
$$

where

$$
\begin{aligned}
& h_{1}=\frac{1}{2 \rho} \frac{\lambda_{T}^{2}}{\left(1+\lambda_{T}^{2} \sigma^{2}\right)^{2}} \\
& h_{2}=\frac{1}{2 \rho} \frac{2 \lambda_{T} \sigma^{2}}{\left(1+\lambda_{T}^{2} \sigma^{2}\right)^{2}}
\end{aligned}
$$

\subsection{A Plackett-Dale Formulation}

Assume that the cumulative distributions of $S_{i}$ and $T_{i}$ are given by $F_{S_{i}}$ and $F_{T_{i}}$. The joint cumulative distribution of both these quantities has been studied by Plackett ${ }^{[28]}$ and takes the form:

$$
F_{T_{i}, S_{i}}= \begin{cases}\frac{1+\left(F_{T_{i}}+F_{S_{i}}\right)\left(\psi_{i}-1\right)-C\left(F_{T_{i}}, F_{S_{i}}, \psi_{i}\right)}{2\left(\psi_{i}-1\right)} & \text { if } \psi_{i} \neq 1 \\ F_{T_{i}} F_{S_{i}} & \text { if } \psi_{i}=1\end{cases}
$$

Wiley StatsRef: Statistics Reference Online, @ 2014-2018 John Wiley \& Sons, Ltd.

This article is $\odot 2018$ John Wiley \& Sons, Ltd.

DOI: 10.1002/9781118445112.stat07623.pub2 
where $\psi_{i}, F_{T_{i}}$, and $F_{S_{i}}$ are the odds ratio (function) and two marginal cumulative distribution functions. Further,

$$
C\left(q_{1}, q_{2}, \psi\right)=\sqrt{\left[1+\left(q_{1}+q_{2}\right)(\psi-1)\right]^{2}+4 \psi(1-\psi) q_{1} q_{2}}
$$

for $0 \leq q_{1}, q_{2} \leq 1$ and $0 \leq \psi<+\infty$.

We can now derive a bivariate Plackett "density" function $G_{i}(t, s)$ for mixed continuous-binary outcomes. Suppose the success probability for $S_{i}$ is denoted by $\pi_{i}$, then we can define $G_{i}(t, s)$ by specifying $G_{i}(t, 0)$ and $G_{i}(t, 1)$ such that they sum to $f_{T_{i}}(t)$. If we define

$$
G_{i}(t, 0)=\frac{\partial F_{T_{i}, S_{i}}(t, 0)}{\partial t}
$$

then this leads to specifying $G_{i}$ by

$$
G_{i}(t, 0)= \begin{cases}\frac{f_{T_{i}}(t)}{2}\left(1-\frac{1+F_{T_{i}}(t)\left(\psi_{i}-1\right)-F_{S_{i}}(s)\left(\psi_{i}+1\right)}{C\left(F_{T_{i}}, 1-\pi_{i}, \psi_{i}\right)}\right) & \text { if } \psi_{i} \neq 1 \\ f_{T_{i}}(t)\left(1-\pi_{i}\right) & \text { if } \psi_{i}=1\end{cases}
$$

and

$$
G_{i}(t, 1)=f_{T_{i}}(t)-G_{i}(t, 0)
$$

In this formulation, we assume $T_{i} \sim N\left(\mu_{i}, \sigma^{2}\right)$, with $\mu_{i}=\mu_{T}+\beta X_{i}$ and $\operatorname{logit}\left(\pi_{i}\right)=\mu_{S}+\alpha X_{i}$ with similar notation as in the probit case. The global odds ratio is assumed to be constant, but this is obviously open to extension. If we write

$$
\theta_{i}=\left(\begin{array}{c}
\mu_{i} \\
\sigma^{2} \\
\pi_{i} \\
\psi
\end{array}\right) \quad \text { and } \quad \eta_{i}=\left(\begin{array}{c}
\mu_{i} \\
\ln \left(\sigma^{2}\right) \\
\operatorname{logit}\left(\pi_{i}\right) \\
\ln (\psi)
\end{array}\right)
$$

estimates of the regression parameters $v=\left(\mu, \beta, \alpha, \ln \sigma^{2}, \ln \psi\right)$ are easily obtained by solving the estimating equations $U(v)=\mathbf{0}$, using a Newton-Raphson iteration scheme, where $U(v)$ is given by

$$
\sum_{i=1}^{n}\left(\frac{\partial \eta_{i}}{\partial v}\right)^{\prime}\left\{\left(\frac{\partial \eta_{i}}{\partial \theta_{i}}\right)^{\prime}\right\}^{-1}\left(\frac{\partial}{\partial \theta_{i}} \ln G_{i}\left(t_{i}, s_{i}\right)\right)
$$

\subsection{A Generalized Linear Mixed Model Formulation}

Following developments of Molenberghs and Verbeke [25, Chapter 24], we can use generalized linear mixed model ${ }^{[2,30]}$ ideas, applied to the outcome vector $Y_{i}$, which in its simplest form can take the form $Y_{i}=\left(S_{i}, T_{i}\right)^{\prime}$. What we need in this context is the decomposition:

$$
Y_{i}=\mu_{i}+\varepsilon_{i}
$$

where

$$
\mu_{i}=\mu_{i}\left(\eta_{i}\right)=h\left(X_{i} \beta+Z_{i} b_{i}\right)
$$

We assume $b_{i} \sim N(\mathbf{0}, D)$. The key relaxing assumption for us is that the components of the inverse link functions $h$ are allowed to change with the nature of the various outcomes in $Y_{i}$. The variance of $\varepsilon_{i}$ depends on the mean-variance links of the various outcomes, and can contain, in addition, a correlation matrix $R_{i}(\alpha)$

Wiley StatsRef: Statistics Reference Online, @ 2014-2018 John Wiley \& Sons, Ltd.

This article is $\odot 2018$ John Wiley \& Sons, Ltd.

DOI: 10.1002/9781118445112.stat07623.pub2 
and overdispersion parameters $\phi_{i}$. When there are no random effects in Equation (15), a marginal model is obtained. We will refer to this as a marginal generalized linear models (MGLM) approach. Reversely, assuming that there are no residual correlations in $R_{i}(\alpha)$, a conditional independence model or purely random effects model results, which is still denoted by GLMM.

Using straightforward derivations, a general first-order approximate expression for the variancecovariance matrix of $Y_{i}$ is:

$$
V_{i}=\operatorname{Var}\left(Y_{i}\right) \simeq \Delta_{i} Z_{i} D Z_{i}^{\prime} \Delta_{i}^{\prime}+\Sigma_{i}
$$

Here,

$$
\Delta_{i}=\left.\left(\frac{\partial \mu_{i}}{\partial \eta_{i}}\right)\right|_{b_{i}=\mathbf{0}}
$$

and

$$
\Sigma_{i} \simeq \Xi_{i}^{1 / 2} A_{i}^{1 / 2} R_{i}(\alpha) A_{i}^{1 / 2} \Xi_{i}^{1 / 2}
$$

with $A_{i}$ a diagonal matrix containing the variances following from the generalized linear model specification of $Y_{i j}$, given the random effects $b_{i}=\mathbf{0}$, that is, with diagonal elements $v\left(\mu_{i j} \mid b_{i}=\mathbf{0}\right)$. Likewise, $\Xi_{i}$ is a diagonal matrix with the overdispersion parameters along the diagonal. When an outcome component is normally distributed, the overdispersion parameter is $\sigma_{i}^{2}$ and the variance function is 1 . For a binary outcome with logit link, we obtain

$$
\mu_{i j}\left(b_{i}=\mathbf{0}\right)\left[1-\mu_{i j}\left(b_{i}=\mathbf{0}\right)\right]
$$

The evaluation under $b_{i}=\mathbf{0}$ derives from a Taylor series expansion of the mean components around $b_{i}=\mathbf{0}$.

When an exponential family specification is used for all components, with canonical link, $\Delta_{i}=A_{i}$ and we can write:

$$
V_{i}=\operatorname{Var}\left(Y_{i}\right) \simeq \Delta_{i} Z_{i} D Z_{i}^{\prime} \Delta_{i}^{\prime}+\Xi_{i}^{1 / 2} \Delta_{i}^{1 / 2} R_{i}(\alpha) \Delta_{i}^{1 / 2} \Xi_{i}^{1 / 2}
$$

Under conditional independence, $R_{i}$ vanishes and

$$
V_{i}=\operatorname{Var}\left(Y_{i}\right)=\Delta_{i} Z_{i} D Z_{i}^{\prime} \Delta_{i}^{\prime}+\Xi_{i}^{1 / 2} \Delta_{i} \Xi_{i}^{1 / 2}
$$

For the setting already considered in Sections 2.1 and 2.2, a suitable version of Equation (14) is:

$$
\left(\begin{array}{c}
S_{i} \\
T_{i}
\end{array}\right)=\left(\begin{array}{c}
\mu_{S}+\lambda b_{i}+\alpha X_{i} \\
\frac{\exp \left[\mu_{T}+b_{i}+\beta X_{i}\right]}{1+\exp \left[\mu_{T}+b_{i}+\beta X_{i}\right]}
\end{array}\right)+\left(\begin{array}{c}
\varepsilon_{S i} \\
\varepsilon_{T i}
\end{array}\right)
$$

Note that we have included a scale parameter $\lambda$ in the continuous component of an otherwise randomintercept model, given that the continuous and binary outcomes are measured on different scales. In this case,

$$
Z_{i}=\left(\begin{array}{c}
\lambda \\
1
\end{array}\right), \quad \Delta_{i}=\left(\begin{array}{cc}
1 & 0 \\
0 & v_{i 2}
\end{array}\right), \quad \Phi=\left(\begin{array}{cc}
\sigma^{2} & 0 \\
0 & 1
\end{array}\right)
$$

with $v_{i 2}=\mu_{i 2}\left(b_{i}=\mathbf{0}\right)\left[1-\mu_{i 2}\left(b_{i}=\mathbf{0}\right)\right]$. Further, let $\rho$ be the correlation between $\varepsilon_{S i}$ and $\varepsilon_{T i}$. Note that $Z_{i}$ is not a design matrix in the strict sense, as it contains an unknown parameter. Nevertheless, it is useful to consider this decomposition.

Wiley StatsRef: Statistics Reference Online, @ 2014-2018 John Wiley \& Sons, Ltd.

This article is $\odot 2018$ John Wiley \& Sons, Ltd.

DOI: 10.1002/9781118445112.stat07623.pub2 


\section{Mixed Outcomes}

This implies that Equation (16) becomes

$$
\begin{aligned}
V_{i} & =\left(\begin{array}{cc}
\lambda^{2} & v_{i 2} \lambda \\
v_{i 2} \lambda & v_{i 2}^{2}
\end{array}\right) \tau^{2}+\left(\begin{array}{cc}
\sigma^{2} & \rho \sigma \sqrt{v_{i 2}} \\
\rho \sigma \sqrt{v_{i 2}} & v_{i 2}
\end{array}\right) \\
& =\left(\begin{array}{cc}
\lambda^{2} \tau^{2}+\sigma^{2} & v_{i 2} \lambda \tau^{2}+\rho \sigma \sqrt{v_{i 2}} \\
v_{i 2} \lambda \tau^{2}+\rho \sigma \sqrt{v_{i 2}} & v_{i 2}^{2} \tau^{2}+v_{i 2}
\end{array}\right)
\end{aligned}
$$

The approximate marginal correlation function derived thereof equals:

$$
\rho(\beta)=\frac{v_{i 2} \lambda \tau^{2}+\rho \sigma \sqrt{v_{i 2}}}{\sqrt{\lambda^{2} \tau^{2}+\sigma^{2}} \sqrt{v_{i 2}^{2} \tau^{2}+v_{i 2}}}
$$

Obviously, Equation (21) depends on the fixed effects through $v_{i 2}$. In the special case of no random effects, the model can be written as:

$$
\left(\begin{array}{c}
S_{i} \\
T_{i}
\end{array}\right)=\left(\begin{array}{c}
\mu_{S}+\alpha X_{i} \\
\frac{\exp \left(\mu_{T}+\beta X_{i}\right)}{1+\exp \left(\mu_{T}+\beta X_{i}\right)}
\end{array}\right)+\left(\begin{array}{c}
\varepsilon_{S i} \\
\varepsilon_{T i}
\end{array}\right)
$$

and Equation (21) simply reduces to $\rho$, by virtue of its fully marginal specification. Under conditional independence, $\rho$ in Equation (20) satisfies $\rho \equiv 0$, and Equation (21) reduces to

$$
\rho(\beta)=\frac{v_{i 2} \lambda \tau^{2}}{\sqrt{\lambda^{2} \tau^{2}+\sigma^{2}} \sqrt{v_{i 2}^{2} \tau^{2}+v_{i 2}}}
$$

somewhat simpler but still a function of the fixed effects.

In case both endpoints are binary, the counterpart to Equation (21) is

$$
\rho(\beta)=\frac{v_{i 1} v_{i 2} \tau^{2}+\rho \sigma \sqrt{v_{i 1} v_{i 2}}}{\sqrt{v_{i 1}^{2} \tau^{2}+v_{i 1}} \sqrt{v_{i 2}^{2} \tau^{2}+v_{i 2}}}
$$

with again a constant correlation $\rho$ when there are no random effects, and when there is no residual correlation:

$$
\rho(\beta)=\frac{v_{i 1} v_{i 2} \tau^{2}}{\sqrt{v_{i 1}^{2} \tau^{2}+v_{i 1}} \sqrt{v_{i 2}^{2} \tau^{2}+v_{i 2}}}
$$

Of course, the earlier calculations can be performed with ease for general random effects design matrices $Z_{i}$ and for more than two components, of arbitrary nature and not just continuous and binary. This is useful, for example, for a fully hierarchical specification such as in Section 3.

In the general model, no full joint distribution needs to be specified, even when we assume the first one to be normally distributed, and the second one to be Bernoulli distributed. We can still leave the specification of the joint moments to the second one, by way of the marginal correlation. A full joint specification would need full bivariate model specification, conditional upon the random effects.

Under conditional independence, the specification of the outcome distributions conditional upon the random effects, together with the normality assumptions made about the random effects, fully specifies the joint distribution.

Wiley StatsRef: Statistics Reference Online, () 2014-2018 John Wiley \& Sons, Ltd. 


\section{Hierarchical Joint Models}

In the previous section, bivariate models have been discussed for the joint analysis of a continuous and a binary outcome. Of course, joint outcomes can be measured repeatedly over time, or might be observed within another hierarchical context, such as clusters. In Section 3.1, a two-stage approach is presented, whereas Section 3.2 discusses fully hierarchical models.

\subsection{Two-Stage Analysis}

In this section, we retain the setting of a binary and a continuous endpoint, measured within a hierarchical setting. Molenberghs et al. ${ }^{[8]}$ used this approach in the context of surrogate marker evaluation. Let $\tilde{S}_{i j}$ be a latent variable of which $S_{i j}$ is a dichotomized version. One option is to consider a two-step analysis. Assume that subject $j$ is measured within trial $i$. For repeated measures, $j$ would refer to time and $i$ to subject.

At the first step, we can assume the following model:

$$
\begin{gathered}
\tilde{S}_{i j}=\mu_{S_{i}}+\alpha_{i} X_{i j}+\varepsilon_{S_{i j}} \\
T_{i j}=\mu_{T_{i}}+\beta_{i} X_{i j}+\varepsilon_{T_{i j}}
\end{gathered}
$$

where $\alpha_{i}$ and $\beta_{i}$ are study-specific effects of treatment $X$ on the endpoints in trial $i, \mu_{S_{i}}$ and $\mu_{T_{i}}$ are trialspecific intercepts, and $\varepsilon_{S_{i}}$ and $\varepsilon_{T_{i}}$ are correlated error terms, assumed to be mean-zero normally distributed with the covariance matrix

$$
\Sigma=\left(\begin{array}{cc}
\frac{1}{\left(1-\rho^{2}\right)} & \frac{\rho \sigma}{\sqrt{1-\rho^{2}}} \\
\frac{\rho \sigma}{\sqrt{1-\rho^{2}}} & \sigma^{2}
\end{array}\right)
$$

In short, we use the probit formulation, described in Section 2.1. Owing to the replication at the study level, we can impose a distribution on the study-specific parameters. At the second stage, we assume

$$
\left(\begin{array}{c}
\mu_{S_{i}} \\
\mu_{T_{i}} \\
\alpha_{i} \\
\beta_{i}
\end{array}\right)=\left(\begin{array}{c}
\mu_{S} \\
\mu_{T} \\
\alpha \\
\beta
\end{array}\right)+\left(\begin{array}{c}
m_{S_{i}} \\
m_{T_{i}} \\
a_{i} \\
b_{i}
\end{array}\right)
$$

where the second term on the right-hand side of Equation (26) is assumed to follow a zero-mean normal distribution with dispersion matrix $\boldsymbol{D}$.

\subsection{Fully Hierarchical Modeling}

We first indicate how the probit-normal and Plackett-Dale models can be generalized to the hierarchical setting. Ample details can be found in Geys et al. ${ }^{[21]}$ and Regan and Catalano ${ }^{[15]}$. Next, the GLMM case will be considered.

\subsubsection{A Probit-Normal formulation}

The model of Section 2.1 can be seen as the basis for this model. While Models (1) and (2) apply to one continuous and one binary outcome, we could equally well consider multiple copies of each and then assume 


\section{Mixed Outcomes}

that the resulting stochastic vector, composed of directly observed and latent outcomes, is normally distributed.

Although this approach is natural and appealing, the problem is the handling of potentially highdimensional probits, and several authors have considered this problem in detail. Regan and Catalano ${ }^{\text {[19] }}$ introduced a mixed outcome probit model that extends a correlated probit model for binary outcomes ${ }^{[20]}$ to incorporate continuous outcomes. These authors consider exchangeability among the continuous outcomes, among the binary outcomes, and between the continuous and binary outcomes.

Regan and Catalano ${ }^{[23]}$ avoided fully specifying the joint distribution of the $n_{i}$ bivariate outcomes on related subjects within unit $i$ by specifying only the marginal distribution of the bivariate outcomes and applying GEE to take correlation into account. Precisely, they fully model the bivariate outcomes for a subject and then apply GEE to accommodate for the correlations between subjects within unit $i$.

\subsubsection{A Plackett-Dale approach}

Likewise, the Plackett-Dale model of Section 2.2 can be embedded in a hierarchical setting. Geys et al. ${ }^{\text {[21] }}$ applied marginal pseudo-likelihood ideas for model fitting. We sketch their approach here.

In Section 2.2, a bivariate density distribution was defined for a joint continuous and binary outcome, by means of Equations (12) and (13). In principle, a $2 n_{i}$-dimensional Plackett-Dale model needs to be specified. Alternatively, progress can be made by solely specifying the bivariate outcomes, just as before, and assembling them into a $(\log )$ pseudolikelihood function:

$$
p \ell=\sum_{i=1}^{N} \sum_{j=1}^{n_{i}} \ln G_{i j}\left(t_{i j}, s_{i j}\right)
$$

where $T_{i j}$ is the continuous outcomes for subject $j$ within unit (study, trial, center, etc.) $i$ and $S_{i j}$ is the binary one. Thus, with this particular choice of pseudolikelihood function, the longitudinal part of the correlation structure is left unspecified. Of course, alternative pseudolikelihood functions can be used as well, depending on which parameters are needed to formulate answers to scientific questions. Sometimes, the correlation structure between outcomes on different subjects within the same unit can be of interest, calling for other types of pseudolikelihood function. Parameter and precision estimation based on Equation (27) is straightforward; details can be found in Molenberghs and Verbeke [25, Chapters 9 and 24].

\subsubsection{A Generalized Linear Mixed Model Formulation}

The developments in Section 2.3 extend straightforwardly to the hierarchical case, including repeated measures, meta-analyses, clustered data, and correlated data. In fact, Model (14) is sufficiently general to generate marginal and random-effects models for such settings. The fixed and random effects structures can be formulated sufficiently generally, so as to cover all of these settings. Of course, when parameters are shared between models for outcomes of different types, care has to be taken to ensure that the models are meaningful. For example, inflation factors might have to be used to share random effects across binary and continuous outcomes, exactly as the parameter $\lambda$ in Equation (19).

Correlations follow in a straightforward manner when purely marginal versions are used. When random effects are involved, correlation structures can be derived from Equation (16) or specific forms derived thereof.

Wiley StatsRef: Statistics Reference Online, @ 2014-2018 John Wiley \& Sons, Ltd. 


\section{Age-Related Macular Degeneration Trial}

These data arise from a randomized multicenter clinical trial comparing an experimental treatment (interferon- $\alpha$ ) to a corresponding placebo in the treatment of patients with age-related macular degeneration. Here, we focus on the comparison between placebo and the highest dose (6 million units daily) of interferon- $\alpha(Z)$, but the full results of this trial have been reported elsewhere ${ }^{[31]}$. Patients with macular degeneration progressively lose vision. In the trial, the patients' visual acuity was assessed at different time points $(4,12,24$, and 52 weeks) through their ability to read lines of letters on standardized vision charts. These charts display lines of five letters of decreasing size, which the patient must read from top (the largest letters) to bottom (the smallest letters). The raw patient's visual acuity is the total number of letters correctly read. In addition, one often refers to each line with at least four letters correctly read as a "line of vision."

Table 1 shows the visual acuity (mean and standard error) by the treatment group at baseline, at 6 months, and at 1 year. The visual acuity can be measured in several ways. First, one can record the number of letters read. Alternatively, dichotomized versions (at least three lines of vision lost at 1 year, or at least two lines of vision lost at 6 months) can be used as well. Therefore, these data will be useful to illustrate methods for the joint modeling of continuous and binary outcomes, with or without taking the longitudinal nature into account. In addition, though there are 190 subjects with both month 6 and month 12 measurements available, the total number of longitudinal profiles is 240, but for only 188 of these have the four follow-up measurements been made.

In these data, the mixed discrete-continuous case arises because the number of letters read can be treated as continuous, while the dichotomization in terms of at least two or three lines of vision lost at 6 and 12 months, respectively, is a binary outcome.

In Section 4.1, a number of bivariate marginal analyses are presented, with bivariate random-effects analyses discussed in Section 4.2. Hierarchical analyses, based on including center as a hierarchy defining variable on the one hand, and repeated measures on each of the binary and continuous outcomes on the other hand, are presented in Section 4.3.

\subsection{Bivariate Marginal Analyses}

First, we consider dichotomized visual acuity at 6 months as the surrogate and (continuous) visual acuity at 12 months as the true endpoint. Dichotomization is achieved by setting a binary variable to 1 if visual acuity at 6 months is larger than the value at baseline and to 0 otherwise. We consider a probit-normal model as in Section 2.1, a Plackett-Dale model as in Section 2.2, and a GLM-based marginal model as in Section 2.3. Of course, the roles of $S_{i}$ and $T_{i}$ are reversed in the corresponding equations, as here the surrogate is assumed to be binary while the true outcome was binary in the earlier sections. For the latter

Table 1. The Age-related Macular Degeneration Trial. Mean (standard error) of visual acuity at baseline, at 6 months, and at 1 year according to the randomized treatment group (placebo vs interferon- $\alpha$ ).

\begin{tabular}{lccr}
\hline Time point & Placebo & Active & Total \\
\hline Baseline & $55.3(1.4)$ & $54.6(1.3)$ & $55.0(1.0)$ \\
$6 \mathrm{mo}$ & $49.3(1.8)$ & $45.5(1.8)$ & $47.5(1.3)$ \\
$1 \mathrm{yr}$ & $44.4(1.8)$ & $39.1(1.9)$ & $42.0(1.3)$ \\
\hline
\end{tabular}


Table 2. Age-Related Macular Degeneration Trial. Bivariate marginal analyses with a binary surrogate and a continuous true endpoint.

\begin{tabular}{|c|c|c|c|c|c|}
\hline \multirow[b]{2}{*}{ Effect } & \multirow[b]{2}{*}{ Par. } & \multirow[b]{2}{*}{ Probit-normal } & \multirow[b]{2}{*}{ Plackett-Dale } & \multicolumn{2}{|c|}{ MGLM } \\
\hline & & & & Logit & Probit \\
\hline \multicolumn{6}{|c|}{ Binary surrogate endpoint } \\
\hline Intercept & $\mu_{S}$ & $0.64(0.20)$ & $0.74(0.19)$ & $1.25(0.24)$ & $0.76(0.14)$ \\
\hline Treatm. eff. & $\alpha$ & $0.39(0.28)$ & $0.45(0.30)$ & $0.40(0.38)$ & $0.23(0.21)$ \\
\hline Overdis. par. & $\phi$ & & & $1.01(0.10)$ & $1.01(0.10)$ \\
\hline \multicolumn{6}{|c|}{ Continuous true endpoint } \\
\hline Intercept & $\mu_{T}$ & $11.04(1.57)$ & $10.89(1.56)$ & $11.04(1.58)$ & $11.04(1.58)$ \\
\hline Treatm. eff. & $\beta$ & $4.12(2.32)$ & $4.02(2.32)$ & $4.12(2.33)$ & $4.12(2.33)$ \\
\hline Standard dev. & $\sigma_{T}$ & $15.95(0.82)$ & $16.04(0.81)$ & & \\
\hline Variance & $\sigma_{T}^{2}$ & $254.4(26.2)$ & $257.3(26.0)$ & $257.0(26.5)$ & $257.0(26.5)$ \\
\hline & & & ciation & & \\
\hline Log odds r. & $\ln \psi$ & $0.14(0.0)$ & $2.85(0.37)$ & $0.02(0.05)$ & $0.02(0.05)$ \\
\hline Odds r. & $\psi$ & & $17.29(6.40)$ & & \\
\hline
\end{tabular}

model, both a logit as well as a probit link is considered for the MGLM. PQL is used as the approximation method. Given the current availability of numerical integration methods, adaptive Gaussian quadrature, as implemented in the SAS procedures GLIMMIX and NLMIXED, is recommended. For the Plackett-Dale model, a logit link is employed for the true endpoint. Parameter estimates (standard errors) are displayed in Table 2.

The correlation between both endpoints is estimated as $\hat{\rho}=0.74$ under the probit model. This parameter is of direct interest in surrogate marker evaluation as it captures the so-called adjusted association ${ }^{[32]}$ or individual-level association ${ }^{[8,33]}$. It also justifies the use of a joint model for both endpoints, rather than considering them separately. This parameter is estimated very precisely and there is apparently a strong correlation between both endpoints. Now, the corresponding correlation under the GLM is quite a bit lower. Although, owing to the use of PQL, there typically is downward bias in the parameter estimates, a more important reason for the difference is that the probit model features the correlation between a pair of latent variables, whereas the GLM captures the correlation between the observable outcomes. The Plackett-Dale model, of course, is based on the use of the odds ratio rather than the correlation as the association parameter. For the binary endpoint, the treatment effect parameters differ somewhat, with the differences in the intercepts a bit larger. The parameter estimates for the continuous endpoint agree much closer.

Let us now switch to the situation of continuous visual acuity at 6 months as a surrogate for the binary indicator for loss of at least 3 lines of vision lost at 1 year. The same models as in Table 2 are considered here too, with of course the roles of the continuous and binary endpoints reversed. Parameter estimates (standard errors) are given in Table 3. Qualitative conclusions agree very closely with their counterparts for the earlier analyses, although there are some quantitative differences. With the probit model, the correlation is $\hat{\rho}=0.81$, but again, for the GLM-based models, they are quite a bit smaller, underscoring once more that the two correlation parameters are not really directly comparable, as the probit (and also Dale) versions describe the correlation of the underlying bivariate latent variable. With the Plackett-Dale model, the odds ratio is estimated to be $\widehat{\psi}=16.93$. As in Table 2, parameter estimates across models agree fairly closely, but the agreement is better for the continuous endpoint than for the binary one.

Wiley StatsRef: Statistics Reference Online, @ 2014-2018 John Wiley \& Sons, Ltd. 
Table 3. Age-Related Macular Degeneration Trial. Bivariate marginal analyses with a continuous surrogate and a binary true endpoint.

\begin{tabular}{|c|c|c|c|c|c|}
\hline \multirow[b]{2}{*}{ Effect } & \multirow[b]{2}{*}{ Par. } & \multirow[b]{2}{*}{ Probit-normal } & \multirow[b]{2}{*}{ Plackett-Dale } & \multicolumn{2}{|c|}{ MGLM } \\
\hline & & & & Logit & Probit \\
\hline \multicolumn{6}{|c|}{ Continuous surrogate endpoint } \\
\hline Intercept & $\mu_{S}$ & $5.53(1.26)$ & $5.89(1.24)$ & $5.53(1.27)$ & $5.53(1.27)$ \\
\hline Treatm. eff. & $\alpha$ & $2.83(1.87)$ & $2.72(1.84)$ & $2.83(1.87)$ & $2.83(1.87)$ \\
\hline Standard dev. & $\sigma_{S}$ & $12.80(0.66)$ & $12.90(0.65)$ & & \\
\hline Variance & $\sigma_{s}^{2}$ & $163.8(16.9)$ & $166.4(16.8)$ & $165.7(17.1)$ & $165.7(17.1)$ \\
\hline \multicolumn{6}{|c|}{ Binary true endpoint } \\
\hline Intercept & $\mu_{T}$ & $-0.36(0.21)$ & $-0.36(0.19)$ & $-0.50(0.20)$ & $-0.31(0.13)$ \\
\hline Treatm. eff. & $\beta$ & $0.60(0.30)$ & $0.58(0.28)$ & $0.66(0.30)$ & $0.41(0.19)$ \\
\hline Overdis. par. & $\phi$ & & & $1.01(0.10)$ & $1.01(0.10)$ \\
\hline Correlation & $\rho$ & $0.81(0.04)$ & 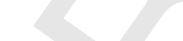 & $0.62(0.04)$ & $0.62(0.04)$ \\
\hline Log odds r. & $\ln \psi$ & & $2.83(0.29)$ & & \\
\hline Odds r. & $\psi$ & & $16.93(4.91)$ & & \\
\hline
\end{tabular}

Of course, one could also analyze both endpoints as binary, or both endpoints as continuous. Although not the theme of the article, it is useful to do so for the sake of comparison. In the first case, a standard probit or Dale model could be used. In the second case, a bivariate normal is the obvious choice. Let us first focus on the situation of two binary outcomes. Buyse and Molenberghs ${ }^{[32]}$ analyzed both binary endpoints using the Dale model with logit links and obtained an odds ratio of $\hat{\psi}=18.53$. Table 4 presents five different analyses of the pair of binary outcomes. First, the Dale model is fitted with both logit and probit links. Second, a marginal linearization-based model with correlated error terms is considered, again with logit and probit links. Third, a bivariate probit model is fitted. Table 4 organizes the models by link function, so that similarities and differences between parameter estimates become more apparent.

Even more so than in the heterogeneous outcome cases, there is close agreement between the intercept and treatment effect parameter estimates for the logit and probit models, respectively. At the same time, there is agreement between the association measures as far as they are comparable, but once again the probit-based correlation is quite a bit higher than the GLM-based correlation, for reasons explained earlier.

Finally, both outcomes can be considered as continuous. Then, the counterparts of all models in Tables 2-4 collapse to a bivariate normal model, and so does the output obtained from virtually all relevant software tools, such as the SAS procedures MIXED, NLMIXED, and GLIMMIX. The results are presented in Table 5. The correlation obtained here is 0.75 . Note that this is closer to the bivariate probit and probit-normal models than to the GLM one. Indeed, we now have a bivariate continuous outcome, which is more informative than a pair of binary outcomes or the joint occurrence of a binary and a continuous outcome. Nevertheless, in all situations do the probit and probit-normal models attempt to describe the association of the underlying pair of normal outcomes, whether or not they are directly observed.

Generally note that, when continuous or binary outcome results are compared across Tables 2-5, whether from a heterogeneous or homogenous model, there is reasonably close agreement, especially within a model family (probit-normal, Plackett-Dale, GLM-based), and especially for treatment effects and association parameters. 
Table 4. Age-Related Macular Degeneration Trial. Bivariate marginal analyses with binary endpoints, based on the Dale model (probit and logit links), the bivariate probit model, and a marginal joint GLM (logit and probit links).

\begin{tabular}{|c|c|c|c|c|}
\hline \multirow[b]{2}{*}{ Effect } & \multirow[b]{2}{*}{ Parameter } & \multicolumn{3}{|c|}{ Logit links } \\
\hline & & & Dale & MGLM \\
\hline \multicolumn{5}{|c|}{ Surrogate endpoint } \\
\hline Intercept & $\mu_{S}$ & & $-0.54(0.20)$ & $-0.54(0.21)$ \\
\hline Treatm. eff. & $\alpha$ & & $0.70(0.30)$ & $0.70(0.30)$ \\
\hline Overdis. par. & $\phi$ & & & $1.01(0.10)$ \\
\hline \multicolumn{5}{|c|}{ True endpoint } \\
\hline Intercept & $\mu_{T}$ & & $-0.50(0.20)$ & $-0.50(0.20)$ \\
\hline Treatm. eff. & $\beta$ & & $0.66(0.30)$ & $0.66(0.30)$ \\
\hline Overdis. par. & $\phi$ & & & $1.01(0.10)$ \\
\hline \multirow{4}{*}{\multicolumn{5}{|c|}{$\begin{array}{l}\text { Correlation } \\
\text { Log odds r. } \\
\text { Odds r. }\end{array}$}} \\
\hline & & & & \\
\hline & & & & \\
\hline & & & & \\
\hline \multirow[b]{2}{*}{ Effect } & \multirow[b]{2}{*}{ Parameter } & \multicolumn{3}{|c|}{ Probit links } \\
\hline & & Biv. probit & Dale & MGLM \\
\hline & & Surrogate endpoint & $\checkmark$ & \\
\hline Intercept & $\mu_{S}$ & $-0.34(0.13)$ & $-0.33(0.13)$ & $-0.33(0.13)$ \\
\hline Treatm. eff. & $\alpha$ & $0.44(0.18)$ & $0.44(0.18)$ & $0.44(0.19)$ \\
\hline Overdis. par. & $\phi$ & & & $1.01(0.10)$ \\
\hline Intercept & $\mu_{T}$ & $-0.31(0.13)$ & $-0.31(0.13)$ & $-0.31(0.13)$ \\
\hline Treatm. eff. & $\beta$ & $0.41(0.18)$ & $0.41(0.18)$ & $0.41(0.19)$ \\
\hline Overdis. par. & $\phi$ & & & $1.01(0.10)$ \\
\hline Correlation & $\rho$ & $0.83(0.05)$ & & $0.62(0.05)$ \\
\hline Log odds r. & $\ln \psi$ & & $2.92(0.38)$ & \\
\hline Odds r. & $\psi$ & & $18.54(7.05)$ & \\
\hline
\end{tabular}

\subsection{Bivariate Random-Effects Analyses}

Although all the abovementioned models are of a marginal type, we can also consider random-effects models. So far, we have considered marginal versions of Equation (14), denoted by MGLM, but we will now switch to the conditional independence model (19) with a scaled random intercept, for the case of a binary and a continuous outcome, a classical random-intercepts logistic regression model when both outcomes are binary, and a random-intercept linear mixed effects model for continuous outcomes. The results are presented in Table 6.

Comparing the continuous-binary case with the results in Table 3, it is clear that fixed effects in the Gaussian model roughly remain the same, but the fixed effects for the binary outcome are larger. A similar inflation is seen in the binary-binary case, at least when numerical integration is used. For PQL, the bias is severe and the parameter estimates are hardly larger than their marginal counterparts in Table 4. In line with general results on the linear mixed model, the estimates in the last column are very close to those in

Wiley StatsRef: Statistics Reference Online, @ 2014-2018 John Wiley \& Sons, Ltd. 
Table 5. Age-Related Macular Degeneration Trial. Bivariate marginal analyses with continuous endpoints, using a bivariate normal model.

\begin{tabular}{lcc}
\hline Effect & Par. & Estimate (s.e.) \\
\hline & Surrogate endpoint & \\
Intercept & $\mu_{S}$ & $5.53(1.27)$ \\
Treatm. eff. & $\alpha$ & $2.83(1.87)$ \\
Standard dev. & $\sigma_{S}$ & $12.87(0.66)$ \\
Variance & $\sigma_{S}^{2}$ & $165.7(17.1)$ \\
& True endpoint & \\
Intercept & $\mu_{T}$ & $11.04(1.58)$ \\
Treatm. eff. & $\beta$ & $4.12(2.33)$ \\
Standard dev. & $\sigma_{T}$ & $16.03(0.83)$ \\
Variance & $\sigma_{T}^{2}$ & $257.0(26.5)$ \\
& Association & \\
Correlation & $\rho$ & $0.75(0.03)$ \\
\hline
\end{tabular}

Table 6. Age-Related Macular Degeneration Trial. Bivariate joint generalized linear mixed model analyses.

\begin{tabular}{|c|c|c|c|c|c|}
\hline \multicolumn{2}{|c|}{$\begin{array}{l}\text { Surrogate endpoint: } \\
\text { True endpoint: } \\
\text { Estimation method: }\end{array}$} & \multirow{2}{*}{\multicolumn{2}{|c|}{$\begin{array}{cc}\text { Cont. } & \text { Binary } \\
\text { Binary } & \text { Binary } \\
\text { PQL } & \text { Num. int. } \\
\end{array}$}} & \multirow[t]{2}{*}{$\begin{array}{c}\text { Binary } \\
\text { Binary } \\
\text { PQL }\end{array}$} & \multirow[t]{2}{*}{$\begin{array}{l}\text { Cont. } \\
\text { Cont. } \\
\text { ML }\end{array}$} \\
\hline Effect & Par. & & & & \\
\hline \multicolumn{6}{|c|}{ Surrogate endpoint parameters } \\
\hline Intercept & $\mu_{\mathrm{S}}$ & $5.53(1.26)$ & $1.42(0.57)$ & $-0.62(0.26)$ & $5.53(1.42)$ \\
\hline Treatm. eff. & $\alpha$ & $2.83(1.86)$ & $-1.84(0.82)$ & $0.81(0.39)$ & $2.83(2.11)$ \\
\hline Standard dev. & $\sigma_{\mathrm{S}}$ & $7.18(1.15)$ & & & \\
\hline Variance & $\sigma_{s}^{2}$ & 51.59 (16.55) & & & \\
\hline Inflation & $\vec{\lambda}$ & $-1.41(1.68)$ & & & \\
\hline \multicolumn{6}{|c|}{ True endpoint parameters } \\
\hline Intercept & $\mu_{T}$ & $1.63(1.94)$ & $1.31(0.56)$ & $-0.57(0.26)$ & $11.04(1.42)$ \\
\hline Treatm. eff. & $\beta$ & $-2.72(3.15)$ & $-1.73(0.81)$ & $0.76(0.39)$ & $4.12(2.11)$ \\
\hline \multicolumn{6}{|c|}{ Common parameters, including association } \\
\hline R.l. std.d. & $\tau$ & $7.50(8.50)$ & $3.81(0.69)$ & $1.95(0.47)$ & $12.41(0.76)$ \\
\hline R.I. var. & $\tau^{2}$ & $56.2(127.4)$ & $14.51(5.28)$ & $3.76(1.82)$ & $154.0(18.8)$ \\
\hline Res. st.d. & $\sigma$ & & & & $7.43(0.38)$ \\
\hline Res. var. & $\sigma^{2}$ & & & & $55.1(15.7)$ \\
\hline Correlation & $\rho$ & & & & 0.74 \\
\hline Corr. (stand.) & $\rho[1]$ & 0.79 & 0.78 & 0.48 & \\
\hline Corr. (exp.) & $\rho[2]$ & 0.78 & 0.70 & 0.46 & \\
\hline
\end{tabular}

Some models lead to a treatment arm-dependent correlation estimate, denoted by "stand" for the standard arm and "exp" for the experimental arm

Table 5, even though the assumption of a constant variance, made here, may be somewhat too simplistic, given that the variances in Table 5 are quite a bit different.

As before, the correlation between both endpoints is of interest. With the exception of the continuous-continuous case, it is somewhat less straightforward to derive. For the continuous-binary case, we can make use of Equation (23) and for the binary-binary case, Equation (24) is the proper 
Table 7. Age-Related Macular Degeneration Trial. Parameter estimates (standard errors) for the full and reduced two-stage fixed effects probit model.

\begin{tabular}{lcrc}
\hline Effect & Parameter & Full & Reduced \\
\hline & & Surrogate endpoint \\
Intercept & $\mu_{S}$ & $1.46(0.68)$ & $0.67(0.15)$ \\
Treatm. eff. & $\alpha$ & $1.10(0.98)$ & $1.75(0.69)$ \\
& & True endpoint & \\
Intercept & $\mu_{T}$ & $11.13(1.69)$ & $11.82(1.00)$ \\
Treatm. eff. & $\beta$ & $4.40(2.94)$ & $3.72(2.38)$ \\
Standard dev. & $\sigma_{T}$ & $11.43(0.60)$ & $13.60(0.71)$ \\
Variance & $\sigma_{T}^{2}$ & $130.6(13.7)$ & $185.0(19.3)$ \\
& & & $0.66(0.07)$ \\
Correlation & $\rho$ & Association &
\end{tabular}

choice. Clearly, the correlation is different between both treatment arms now, given the dependence of the correlation function on the fixed effects. However, in this case, the difference is negligible. However, the poverty of the PQL approximation is shown, not only in the fixed effect and variance component estimates, but also in the correlation parameters. For the others, irrespective of the nature of the outcomes, the results are very close to their marginal counterparts in Tables $2-5$, which is reassuring.

It is worthwhile to note that the parameters in the continuous-binary case are identifiable, but due to the nonlinearity of the model, induced by the factor $\lambda$, care has to be taken in monitoring the convergence process. Having said this, the effect is most clearly seen on the binary outcome fixed effects, and not quite as much on the continuous outcome parameters.

\subsection{Hierarchical Analyses}

Let us now switch attention to the hierarchical case. First, we observe that the trial is of a multicenter type. It is natural to consider the center in which the patients were treated as the unit of analysis. A total of 36 centers were thus available for analysis, with a number of individual patients per center ranging from 2 to 18 . We analyze the situation where dichotomized visual acuity at 6 months acts as a surrogate for the continuous visual acuity at 12 months. A two-stage approach is followed. Table 7 shows the parameter estimates for the hierarchical probit model (Section 3.1). Two versions are considered, with trial-specific treatment effects on the one hand (reduced model) and with trial-specific intercepts and treatment effects on the other hand (full model).

The correlation, obtained from the full model, is similar to the ones obtained from the bivariate analyses. When the reduced model is employed, the correlation is quite a bit smaller.

Of course, also fully hierarchical models can be fitted. For example, the hierarchical probit or Plackett-Dale models can be used. Applications of these models can be found in Regan and Catalano ${ }^{[15]}$. Also, the joint generalized linear mixed effects model of Section 2.3 can be used for hierarchical analyses. Although we have focused so far on outcomes at 6 months and 1 year, we will now also consider the intermediate endpoints at 4 and 12 weeks as well. Thus, we have two repeated sequences of four components each, one binary and one continuous. The binary outcomes are dichotomizations of the number of letters lost as negative versus nonnegative. We consider on the one hand a marginal model, with fully unstructured $8 \times 8$ variance-covariance matrix and a conditional independence random-intercepts model on the other hand. Parameter estimates are presented in Table 8.

Wiley StatsRef: Statistics Reference Online, @ 2014-2018 John Wiley \& Sons, Ltd. 
Table 8. Age-Related Macular Degeneration Trial. Hierarchical models for joint longitudinal continuous and binary visual acuity sequences.

\begin{tabular}{|c|c|c|c|}
\hline Effect & Parameter & Marginal & Random Int. \\
\hline \multicolumn{4}{|c|}{ Continuous sequence } \\
\hline Intercept 4 & $\beta_{11}$ & $-3.26(0.77 ; 0.81)$ & $-3.27(1.30)$ \\
\hline Intercept 12 & $\beta_{21}$ & $-4.62(1.14: 1.07)$ & $-4.62(1.29)$ \\
\hline Intercept 24 & $\beta_{31}$ & $-8.37(1.38 ; 1.26)$ & $-8.37(1.29)$ \\
\hline Intercept 52 & $\beta_{41}$ & $-15.16(1.72 ; 1.64)$ & $-15.16(1.29)$ \\
\hline Treatment eff. 4 & $\beta_{12}$ & $2.31(1.05 ; 1.05)$ & $2.38(1.76)$ \\
\hline Treatment eff. 12 & $\beta_{22}$ & $2.34(1.54 ; 1.52)$ & $2.34(1.76)$ \\
\hline Treatment eff. 24 & $\beta_{32}$ & $2.83(1.87 ; 1.84)$ & $2.83(1.76)$ \\
\hline Treatment eff. 52 & $\beta_{42}$ & $4.12(2.33 ; 2.31)$ & $4.12(1.76)$ \\
\hline Res. st. deviation & $\sigma$ & & $8.21(0.23)$ \\
\hline Res. variance & $\sigma^{2}$ & & $67.45(3.81)$ \\
\hline Inflation & $\lambda$ & & $-3.32(0.34)$ \\
\hline \multicolumn{4}{|c|}{ Binary sequence } \\
\hline Intercept 4 & $\beta_{11}$ & $-1.02(0.24 ; 0.24)$ & $-2.02(0.46)$ \\
\hline Intercept 12 & $\beta_{21}$ & $-0.91(0.24 ; 0.24)$ & $-1.81(0.45)$ \\
\hline Intercept 24 & $\beta_{31}$ & $-1.15(0.25 ; 0.25)$ & $-2.24(0.47)$ \\
\hline Intercept 52 & $\beta_{41}$ & $-1.65(0.29 ; 0.29)$ & $-3.11(0.52)$ \\
\hline Treatment eff. 4 & $\beta_{12}$ & $0.40(0.32 ; 0.32)$ & $0.66(0.59)$ \\
\hline Treatment eff. 12 & $\beta_{22}$ & $0.54(0.31: 0.31)$ & $0.93(0.58)$ \\
\hline Treatment eff. 24 & $\beta_{32}$ & $0.52(0.33 ; 0.32)$ & $0.88(0.60)$ \\
\hline Treatment eff. 52 & $\beta_{42}$ & $0.40(0.38 ; 0.38)$ & $0.62(0.64)$ \\
\hline \multicolumn{4}{|c|}{ Common parameters } \\
\hline R.I. st. deviation & $\tau$ & & $2.66(0.29)$ \\
\hline R.I. variance & $\tau^{2}$ & & 7.07 (1.64) \\
\hline
\end{tabular}

The marginal model-based and empirically corrected standard errors are presented.

Model parameters here are better identifiable than their counterparts from the bivariate models, even though care is still needed when selecting starting values. Every possible pair of outcomes in the marginal model has its own correlation coefficient (not shown), whereas in the random-effects model, they follow from the fixed effects and variance components, as was illustrated in Section 4.2, based on expressions such as Equations (21), (23), (24), and (25).

\section{Mixed Outcomes in Risk Assessment}

A common example where joint measurements of continuous and discrete outcomes are encountered is a developmental toxicity study, which typically involves exposing pregnant animals (rats and mice) to an environmental agent during major organogenesis. An overview is given in ${ }^{[34]}$. The dams are sacrificed before normal delivery, at which time the uterus is removed and thoroughly examined. Among viable fetuses, the incidence of any malformation (binary data) and reductions in fetal weight (continuous data) are typically of primary concern, as both have been found to be sensitive indicators of a toxic effect ${ }^{[35]}$. This motivates the formulation of a joint distribution with mixed continuous and discrete outcomes. This is not standard, however, especially as the correlation between pups within each dam's litter - called the litter effect - needs to be recognized. The need to account for clustering of the offspring's responses within litters has long been established ${ }^{[36,37]}$.

Wiley StatsRef: Statistics Reference Online, @ 2014-2018 John Wiley \& Sons, Ltd.

This article is $\odot 2018$ John Wiley \& Sons, Ltd.

DOI: 10.1002/9781118445112.stat07623.pub2 


\section{Mixed Outcomes}

In early developmental toxicity studies, a dose-response model was often characterized for each of the two outcomes (weight and malformation) separately, using appropriate methods to account for litter effects. In risk assessment, for example, the scientists or regulator wishes to estimate a low-risk dose (or exposure) level of the environmental toxin in humans, based on experimental data from laboratory animals (see Cross-Species Extrapolation). For such purposes, the outcome that appears to be most sensitive to the exposure based on the animals' dose-response patterns becomes the focus for assessing risk. However, because these outcomes are correlated ${ }^{[38,39]}$, jointly modeling the outcomes and using the bivariate outcome as a basis for risk assessment is more appropriate. Indeed, ignoring the intrafetus correlation may lead to anticonservative results, because protection against the most sensitive effect assures only partial protection against both developmental outcomes. This motivates the use of a joint model for mixed continuous and discrete outcomes.

\subsection{Models Based on Factorization}

The factorization approach for multiple outcomes applies a conditioning argument to the joint structure of the data. One then factors the joint distribution into a marginal component and a conditional component, where the conditioning is done on either the discrete or continuous outcomes.

One of the first models based on factorization for mixed continuous and discrete outcomes was the general location model, described by Olkin and Tate ${ }^{[12]}$. They factored the joint likelihood of categorical and continuous variables in terms of a marginal distribution of the categorical component and the conditional distribution of the continuous component, given the categorical component. The former is taken as a multinomial distribution, and the latter as a conditional multivariate normal. For more information on this model see Schafer ${ }^{[6]}$. This approach is also used in a variety of additional, unclustered multivariate settings ${ }^{[4,40]}$, including various other factorization methods and tests for independence ${ }^{[2,3]}$.

In the clustered setting of a developmental toxicity study ${ }^{[16,17,41,42]}$, the choice of conditioning is made primarily for statistical convenience rather than biological rationale, as relatively little is understood about the biological mechanisms of developmental toxicity. Here, we summarize some factorization methods for developmental toxicity data.

Catalano and Ryan ${ }^{[16]}$ note that latent variable models provide a useful and intuitive way to motivate the distribution of the discrete outcome. Such models presuppose the existence of an unobservable, normally distributed random variable underlying the binary outcome. The binary event is then assumed to occur if the latent variable exceeds some threshold value. This notion of latent variables has much appeal for toxicologists, because it provides a natural and intuitive framework for the biological mechanism leading to adverse events such as malformation. The model is then parameterized, so that the joint distribution is the product of the marginal distribution of the continuous response and the conditional distribution of the binary response given the continuous one. The marginal distribution of the continuous response is related to covariates using, for example, a linear link function $W_{i k}=\alpha_{0}+\alpha_{1} d_{i}$, where $W_{i k}$ is the weight outcome for the $k$ th fetus in the $i$ th cluster, $d_{i}$ the corresponding dose level, and the parameters $\alpha$ the regression parameters. For the conditional distribution, Catalano and Ryan employ a probit model:

$$
\mathrm{E}\left(M_{i k} \mid \mathbf{W}_{i}\right)=\Phi\left[\beta_{0}+\beta_{1} d_{i}+\beta_{2} \bar{e}_{w ; i}+\beta_{3}\left(e_{w ; i k}-\bar{e}_{w ; i}\right)\right]
$$

where $\Phi(z)$ is the cumulative distribution function of a standard normal variate. In this formulation, $M_{i k}$ stands for the malformation outcome for the $k$ th fetus in the $i$ th cluster, $W_{i}$ is the vector of weight outcomes in the $i$ th cluster, $\boldsymbol{\beta}$ is a vector of regression parameters, $\bar{e}_{w ; i}=\bar{W}_{i}-\left(\alpha_{0}+\alpha_{1} d_{i}\right)$, and $e_{w ; i k}=$ $W_{i k}-\left(\alpha_{0}+\alpha_{1} d_{i}\right)$. Hence, a fetus with a negative value for $\bar{e}_{w ; i}$ (average litter weight lower than expected) or a negative value for $e_{w ; i k}$ (weight smaller than average litter weight) will have a higher probability of

Wiley StatsRef: Statistics Reference Online, @ 2014-2018 John Wiley \& Sons, Ltd. 
malformation. The dependence on the weight residuals further induces the dependence between weight and malformation. The parameter estimation then proceeds in two steps. In the first step, fit a correlated regression of fetal weights on dose and possibly other covariates. In the second step, fit a correlated probit regression of malformation, given weight, on dose using generalized estimating equations ${ }^{[22]}$. Owing to the nonlinearity of the link function relating the conditional mean of the binary response to the covariates, the regression parameters in the probit model of Catalano and Ryan ${ }^{[16]}$ have no direct marginal interpretation. Furthermore, if the model for the mean has been correctly specified, but the model for the association between the binary and continuous outcomes is misspecified, the regression parameters in the probit model are not consistent. The lack of marginal interpretation and lack of robustness may be considered unattractive features of this approach. Important advantages, however, are that the model is readily extended to allow for clustering and that the $\beta$-parameters corresponding to the residuals lend insight into the appropriateness of the assumed covariance structure of the latent variable distribution.

Fitzmaurice and Laird ${ }^{[17]}$ circumvent the difficulties in the approach of Catalano and Ryan ${ }^{[16]}$. They extend the general location model of Olkin and Tate ${ }^{[12]}$ and factor the joint distribution as the product of a marginal Bernoulli distribution for the discrete response and a conditional Gaussian (normal) distribution for the continuous response, given the discrete one. Here also, the mean of the distribution of the continuous response given the discrete outcome is a function of the residual from the discrete model. Under independence, the method yields maximum likelihood estimates of the marginal means that are robust to misspecification of the association between the binary and continuous response. Fitzmaurice and Laird also consider an extension of their model that allows for clustering. Using GEEs, they avoid the computational complexity of maximum likelihood in this more elaborate setting. A conceptual difficulty with their model is the interpretation of the parameters, however, which depend on the cluster size.

Another approach that conditions on the continuous outcome uses conditional Gaussian chain models in which fetal weight is modeled as a function of dose and is conditional on litter size, and then malformation is modeled as a function of dose and is conditional on both fetal weight and litter size ${ }^{[42]}$. Ahn and Chen ${ }^{[41]}$ propose a similar tree-structured logistic regression model that incorporates fetal weight. Other models that condition on the discrete outcomes can be derived by considering different marginal distributions of the binary outcomes in combination with a multivariate normal distribution for the continuous responses, conditional on the binary ones.

A drawback of models based on factorization is that they may be difficult to apply for quantitative risk assessment. While taking into account the dependence between weight and malformation, they do not provide an estimate of the intrafetus correlation. Thus, an expression for the joint probability that a fetus is affected (i.e., malformed and/or of low birth weight) is difficult to specify. Catalano et al. ${ }^{[43]}$ used a factorization model for quantitative risk assessment, in which direct estimation of the intrafetus correlation is avoided through an approximation using a conditioning argument. To overcome this problem, one needs joint models that directly incorporate the correlation between outcomes. Thus, a desirable model should have three properties: it should (i) allow separate dose-response functions for each component of the bivariate outcome, (ii) account for the association due to clustering within litters, and (iii) estimate the bivariate intrafetus association.

\subsection{Direct Joint Modeling}

Of primary interest in developmental toxicity studies is the determination of the environmental agent's simultaneous effects on fetal weight and malformation incidence, as both are important measures of teratogenicity (see Teratology). Traditional methods for analysis evaluate the outcomes separately using the methods for clustered data discussed earlier. The endpoints are correlated, however, so a more efficient analysis of the bivariate outcome may be obtained by modeling the outcomes jointly. Joint models allow the

Wiley StatsRef: Statistics Reference Online, @ 2014-2018 John Wiley \& Sons, Ltd.

This article is $\odot 2018$ John Wiley \& Sons, Ltd.

DOI: 10.1002/9781118445112.stat07623.pub2 
outcomes to have different relationships with dose, yet incorporate the correlation between the outcomes. These are still subject to further statistical research. Some proposals are discussed next.

Regan and Catalano ${ }^{[19]}$ introduced a probit approach, based on the Ochi and Prentice ${ }^{[20]}$ model for equicorrelated binary data. They assumed an underlying continuous variable for each binary outcome. Hence, the joint distribution of the vector of weight and latent malformation outcomes can be assumed to follow a multivariate normal distribution. The malformation outcomes are then discretized as in a probit model. Their full likelihood approach can easily be used for quantitative risk assessment and model inspection. It provides marginal dose-response models for each outcome and allows for the estimation of the joint risk to a fetus due to malformation and low birth weight. A difficulty, however, is that the tractability of the likelihood limits their model to the assumption of complete exchangeability among the malformation and weight outcomes within a litter, which may not be true. Fortunately, this problem can be avoided using the GEE methodology ${ }^{[23]}$. As in quantitative risk assessment, clustering is a nuisance, Regan and Catalano ${ }^{[23]}$ argue that one can avoid fully specifying the distribution within a litter by specifying only the marginal distribution of the bivariate outcome and using GEEs to account for correlations due to clustering. Their GEEs are derived from the marginal distribution of the bivariate outcome, which defines the mean and association parameters of interest. The association parameters are estimated via second-order GEEs. The nuisance parameters that account for clustering are estimated via the method of moments, as in first-order GEEs. Both approaches share the attractive feature that they maintain marginal doseresponse interpretations for the continuous and binary outcomes separately, while at the same time use the correlation of the underlying multi- or bivariate normal distribution to characterize the intrafetus correlation.

Molenberghs et al. ${ }^{[8]}$ use Plackett's ${ }^{[28]}$ latent variable approach to model independent bivariate endpoints in which one component is continuous and the other is binary. The Plackett distribution provides an alternative to frequently used normal latent variables. Its main advantages are the flexibility with which the marginal densities can be chosen (normal, logistic, etc.) and its use of the familiar odds ratio to measure association (providing an alternative to the correlation). Geys et al. ${ }^{[21]}$ extended this method to allow for within-cluster association; specification of the full likelihood was avoided using the pseudo-likelihood methodology. An advantage of their model is that it can estimate the joint risk to a fetus and so apply in quantitative risk assessment. In addition, there is a close connection of the pseudolikelihood function with the likelihood function, which enabled ${ }^{[44]}$ to construct pseudolikelihood ratio test statistics for assessing model adequacy.

\section{High-Dimensional Data}

Thus far, it has been discussed how multiple sequences of repeated measurements can be jointly analyzed. The examples given there considered joint modeling of two (longitudinal) outcomes only. Here, we will extend this to (much) higher dimensions. The motivation for joint modeling, potentially of mixed outcomes, will remain the same. In some cases, joint modeling is required because the association structure between the outcomes is of interest. For example, one may be interested in studying how the association between outcomes evolves over time or how outcome-specific evolutions are related to each other ${ }^{[45]}$. In other cases, joint modeling is needed to be able to draw joint inferences about the different outcomes.

An example where joint modeling of many longitudinal outcomes has proven useful can be found in Fieuws et al. ${ }^{[46]}$, where longitudinally measured hearing thresholds were jointly analyzed, for the left ear and for the right ear, and for 11 different frequencies. This yielded a total of 22 longitudinal sequences per subject.

Wiley StatsRef: Statistics Reference Online, @ 2014-2018 John Wiley \& Sons, Ltd. 
The possibly high dimension raises at least two additional problems, in addition to the issues discussed in earlier sections. First, some of the models often used for the joint analysis of two longitudinal sequences are less applicable for higher dimensions. For example, when using conditional models (Section 1), only two possibilities for the conditioning are possible in the case of two outcomes only: The first outcome can be modeled conditionally on the second, or vice versa. With (much) higher dimensions, (many) more possible conditioning strategies are possible, all yielding different models, of which parameters have different interpretations. Moreover, several of the research questions that require joint modeling are phrased in terms of the parameters in each of the univariate longitudinal models (i.e., longitudinal models for each repeated outcome separately), as was the case in the examples given earlier. Also, the models that are available for two outcomes often exploit the specific nature of those two outcomes, making extensions to higher dimensions far from straightforward. For example, the multivariate vector of responses may consist of outcomes of (many) different types, all requiring different models such as linear mixed models, GLMMs, and perhaps even nonlinear mixed models. Second, even if a plausible joint model can be formulated, fitting of these high-dimensional models can become very cumbersome, unless under unrealistically strong assumptions.

In this section, we will focus on the random-effects approach, which can be viewed as an extension of the models discussed in Section 3. The model will be introduced in Section 6.1. Many applications of this type of joint models can be found in the statistical literature. For example, the approach has been used in a nonlongitudinal setting to validate surrogate endpoints in meta-analyses ${ }^{[33,47]}$ or to model multivariate clustered data ${ }^{[48]}$. Gueorguieva ${ }^{[49]}$ used the approach for the joint modeling of a continuous and a binary outcome measure in a developmental toxicity study on mice. Also in a longitudinal setting, Chakraborty et al. ${ }^{[50]}$ obtained estimates of the correlation between blood and semen HIV-1 RNA using a joint randomeffects model. Other examples with longitudinal studies can be found in MacCallum et al. ${ }^{[51]}$, Thiébaut et al. ${ }^{[52]}$, and Shah et al. ${ }^{[53]}$. All these examples refer to situations where the number of different outcomes is (very) low. Although the model formulation can be done irrespective of the number of outcomes to be modeled jointly, standard fitting procedures, such as the maximum likelihood estimation, will only be feasible when the dimension is sufficiently low (typically dimension two or three, at most). Therefore, Section 6.2 presents a model-fitting procedure which is applicable, irrespective of the dimensionality of the problem, and explains how inferences can be obtained for all parameters in the joint model. Finally, Section 6.3 applies the methodology for the joint analysis of seven sets of questionnaires, each consisting of a number of binary outcomes. Other examples, simulation results, and more details on the models as well as on estimation and inference can be found in Fieuws et al. ${ }^{[46]}$, Fieuws and Verbeke ${ }^{[54]}$.

In what follows, models for a single longitudinal outcome are called "univariate" models, although they are, strictly speaking, multivariate models as they model a vector of repeated measurements, but all with the same outcome. Similarly, we will use the terminology "bivariate" and "multivariate" models to indicate joint longitudinal models for two or more outcomes, respectively.

\subsection{Joint Mixed Model}

A flexible joint model that meets the requirements discussed earlier can be obtained by modeling each outcome separately using a mixed model (linear, generalized linear, or nonlinear), by assuming that, conditionally on these random effects, the different outcomes are independent, and by imposing a joint multivariate distribution on the vector of all random effects. This approach has many advantages and is applicable in a wide variety of situations. First, the data can be highly unbalanced. For example, it is not necessary that all outcomes are measured at the same time points. Moreover, the approach is applicable for combining linear mixed models, nonlinear mixed models, or GLMMs. The procedure also allows the combination of different types of mixed models, such as a GLMM for a discrete outcome and a nonlinear mixed model for a continuous outcome. 


\section{Mixed Outcomes}

Let $m$ be the dimension of the problem, that is, the number of outcomes that need to be modeled jointly. Further, let $Y_{r i j}$ denote the $j$ th measurement taken on the $i$ th subject, for the $r$ th outcome, $i=1, \ldots, N$, $r=1, \ldots, m$, and $j=1, \ldots, n_{r i}$. Note that we do not assume that the same number of measurements is available for all subjects, nor for all outcomes. Let $Y_{r i}$ be the vector of $n_{r i}$ measurements taken on subject $i$, for outcome $r$. Our model assumes that each $Y_{r i}$ satisfies a mixed model. Let $f_{r i}\left(y_{r i} \mid b_{r i}, \theta_{r}\right)$ be the density of $Y_{r i}$, conditional on a $q_{r}$-dimensional vector $b_{r i}$ of random effects for the $r$ th outcome on subject $i$. The vector $\theta_{r}$ contains all fixed effects and possibly also a scale parameter needed in the model for the $r$ th outcome. Note that we do not assume the same type of model for all outcomes: A combination of linear, generalized linear, and nonlinear mixed models is possible, for mixed outcome sequences. It is also not assumed that the same number $q_{r}$ of random effects is used for all $m$ outcomes.

In most applications, it will be assumed that, conditionally on the random effects $b_{1 i}, b_{2 i}, \ldots, b_{m i}$, the $m$ outcomes $Y_{1 i}, Y_{2 i}, \ldots, Y_{m i}$ are independent. Extensions of this assumption can be found in Section 3 in the context of surrogate markers, or in Fieuws et al. ${ }^{[46]}$ in the analysis of the 22 longitudinal sequences of hearing thresholds. Finally, the model is completed by assuming that the vector $b_{i}$ of all random effects for subject $i$ is multivariate normal with mean zero and covariance $D$, that is,

$$
b_{i}=\left(\begin{array}{l}
b_{1 i} \\
b_{2 i} \\
\vdots \\
b_{m i}
\end{array}\right) \sim N\left[\left(\begin{array}{l}
0 \\
0 \\
\vdots \\
0
\end{array}\right),\left(\begin{array}{cccc}
D_{11} & D_{12} & \cdots & D_{1 m} \\
D_{21} & D_{22} & \cdots & D_{2 m} \\
\vdots & \vdots & \ddots & \vdots \\
D_{m 1} & D_{m 2} & \cdots & D_{m m}
\end{array}\right)\right]
$$

The matrices $D_{r s}$ represent the covariances between $b_{r i}$ and $b_{s i}, r, s=1, \ldots, m$. Finally, $D$ is the matrix with blocks $D_{r s}$ as entries.

A special case of the abovementioned model is the so-called shared-parameter model, which assumes the same set of random effects for all outcomes. An example of this is Equation (19), where, in the context of surrogate marker evaluation, a random intercept $b_{i}$ was used simultaneously in the model for the surrogate outcome as well as in the model for the true outcome. This can clearly be obtained as a special case of the abovementioned model by assuming a perfect correlation between some of the random effects. The advantage of such shared-parameter models is the relatively low dimension of the random-effects distribution, when compared to the abovementioned model. The dimension of the random effects in shared-parameter models does not increase with the number of outcomes to be modeled. In the abovementioned model, each new outcome added to the model introduces new random effects, thereby increasing the dimension of $b_{i}$. Although the shared-parameter models can be reasonably easily fitted using standard software, this is no longer the case for the model considered here. Estimation and inference under the abovementioned model will require specific procedures, which will be discussed in Section 6.2. A disadvantage of the shared-parameter model is that it is based on much stronger assumptions about the association between the outcomes, which may not be valid, especially in high-dimensional settings as considered in this section.

Note also that, joining valid univariate mixed models does not necessarily lead to a correct joint model. Fieuws and Verbeke ${ }^{[45]}$ illustrate this in the context of linear mixed models for two continuous outcomes. It is shown how the joint model may imply association structures between the two sets of longitudinal profiles that may strongly depend on the actual parameterization of the individual models and that are not necessarily valid.

Estimation and inference will be based on the marginal model for the vector $Y_{i}$ of all measurements for subject $i$. Assuming independence of the outcomes conditionally on the vector $b_{i}$ of random effects, the $\log$-likelihood contribution for subject $i$ equals

$$
\ell_{i}\left(y_{1 i}, y_{2 i}, \ldots, y_{m i} \mid \Psi^{*}\right)=\ln \int \prod_{r=1}^{m} f_{r i}\left(y_{r i} \mid b_{r i}, \theta_{r}\right) f\left(b_{i} \mid D\right) \mathrm{d} b_{i}
$$

Wiley StatsRef: Statistics Reference Online, @ 2014-2018 John Wiley \& Sons, Ltd. 
in which all parameters present in the joint model (fixed effects parameters as well as covariance parameters) have been combined into the vector $\Psi^{*}$.

Clearly, Equation (28) shows that the joint model can be interpreted as one mixed effects model, with conditional density

$$
f_{i}\left(y_{i} \mid b_{i}\right)=\prod_{r=1}^{m} f_{r i}\left(y_{r i} \mid b_{r i}, \theta_{r}\right)
$$

and with random effect $b_{i}$. Hence, fitting of the model can, strictly speaking, be based on standard methods and standard software, available for fitting mixed models in general. However, computational problems will arise as the dimension of the random-effects vector $b_{i}$ in the joint model increases. For example, reconsider the hearing thresholds mentioned earlier. If each of the 22 outcomes is modeled by way of a linear mixed model with random intercepts and random slopes for the time evolution, then the resulting joint model contains $22 \times 2=44$ random effects, resulting in a 44-dimensional matrix $D$ that contains 990 unknown parameters. Even in this case of linear models for continuous data, where the marginal likelihood can be calculated analytically, standard maximization algorithms are no longer sufficient to maximize this marginal likelihood with respect to these many parameters. Moreover, when approximation methods are needed in the calculation of the likelihood, as is the case for generalized or nonlinear mixed models, maximizing the joint likelihood becomes completely impossible using optimization techniques currently implemented for single outcomes. In Section 6.2, we will describe how estimates and inferences for all parameters can be obtained from pairwise fitting of the model, that is, from separately fitting the implied joint model for each pair of outcomes.

\subsection{Model Fitting and Inference}

The general idea behind the pairwise fitting approach is straightforward. Instead of maximizing the likelihood of the full joint model presented in the previous section, all pairwise bivariate models will be fitted separately in a first step. Note the similarity between the pairwise approach used here and the pairwise pseudo-likelihood approach used in Section 3. In a second step, the parameters obtained by fitting the pairwise models will be combined to obtain one single estimate for each parameter in the full joint model.

\subsubsection{Pairwise fitting}

The parameters in each univariate model can be estimated by fitting a model for that specific response only. Hence, the only parameters that cannot be estimated by fitting the univariate models are the parameters needed to model the association between the different outcomes. In the model introduced in Section 6.1, these are the parameters in the matrices $D_{r s}, r \neq s$. However, estimation of these parameters does not necessarily require fitting of the complete joint model for all outcomes, it is sufficient to fit all $m(m-1) / 2$ bivariate models, that is, all joint models for all possible pairs

$$
\left(Y_{1}, Y_{2}\right),\left(Y_{1}, Y_{3}\right), \ldots,\left(Y_{1}, Y_{m}\right),\left(Y_{2}, Y_{3}\right), \ldots,\left(Y_{2}, Y_{m}\right), \ldots,\left(Y_{m-1}, Y_{m}\right)
$$

of the outcomes $Y_{1}, Y_{2}, \ldots, Y_{m}$. Let the log-likelihood function corresponding to the pair $(r, s)$ be denoted by $\ell\left(y_{r}, y_{s} \mid \Psi_{r s}\right)$. The vector $\Psi_{r s}$ contains all parameters in the bivariate model for pair $(r, s)$, that is, the parameters in each of the univariate models, as well as the parameters in $D_{r s}$.

Let $\Psi$ now be the stacked vector combining all $m(m-1) / 2$ pair-specific parameter vectors $\Psi_{r s}$. Estimates for the elements in $\Psi$ are obtained by maximizing each of the $m(m-1) / 2 \log$-likelihoods $\ell\left(y_{r}, y_{s} \mid \Psi_{r s}\right)$ separately. It is important to realize that the parameter vectors $\Psi$ and $\Psi^{*}$ are not equivalent. Indeed, some 
parameters in $\Psi^{*}$ will have a single counterpart in $\Psi$, for example, the parameters in $D_{r s}, r \neq s$, representing covariances between random effects from different outcomes. Other elements in $\Psi^{*}$ will have multiple counterparts in $\Psi$, for example, the parameters in $D_{r r}$, representing variances and covariances of random effects from the same outcome. In the latter case, a single estimate for the corresponding parameter in $\Psi^{*}$ is obtained by averaging all corresponding pair-specific estimates in $\hat{\Psi}$. Standard errors of the so-obtained estimates clearly cannot be obtained from averaging standard errors or variances. Indeed, the variability amongst the pair-specific estimates needs to be taken into account. Furthermore, two pair-specific estimates corresponding to two pairwise models with a common outcome are based on overlapping information and hence correlated. This correlation should also be accounted for in the sampling variability of the combined estimates in $\hat{\Psi}^{*}$. In the remainder of this section, we will use pseudolikelihood ideas to obtain standard errors for the estimates, first in $\hat{\Psi}$, afterward in $\hat{\Psi}^{*}$.

\subsubsection{Inference for $\Psi$}

Fitting all bivariate models is equivalent to maximizing the function

$$
\begin{aligned}
p \ell(\Psi) & \equiv p \ell\left(y_{1 i}, y_{2 i}, \ldots, y_{m i} \mid \Psi\right) \\
& =\sum_{r<s} \ell\left(Y_{r}, Y_{s} \mid \Psi_{r s}\right)
\end{aligned}
$$

ignoring the fact that some of the vectors $\Psi_{r s}$ have common elements, that is, assuming that all vectors $\Psi_{r s}$ are completely distinct. This application of the pseudolikelihood methodology is different from most other applications in the sense that the same parameter vector is usually present in the different parts of the pseudolikelihood function. Here, the set of parameters in $\Psi_{r s}$ is treated pair-specific, which allows separate maximization of each term in the pseudo-log-likelihood function (Equation (29)). In Section 6.2.3, we will account for the fact that $\Psi_{r s}$ and $\Psi_{r s^{\prime}}, s \neq s^{\prime}$, are not completely distinct, as they share the parameters referring to the $r$ th outcome.

Because the pairwise approach fits within the pseudolikelihood framework, an asymptotic multivariate normal distribution for $\hat{\Psi}$ can be derived, using general pseudolikelihood theory ${ }^{[25]}$.

More specifically, we have that $\hat{\Psi}$ asymptotically satisfies

$$
\sqrt{N}(\widehat{\Psi}-\Psi) \approx N\left(0, I_{0}^{-1} I_{1} I_{0}^{-1}\right)
$$

in which $I_{0}^{-1} I_{1} I_{0}^{-1}$ is a "sandwich-type" robust variance estimator, and where $I_{0}$ and $I_{1}$ can be constructed using first- and second-order derivatives of the components in Equation (29). Strictly speaking, $I_{0}$ and $I_{1}$ depend on the unknown parameters in $\Psi$, but these are traditionally replaced by their estimates in $\hat{\Psi}$.

\subsubsection{Combining information: inference for $\Psi^{*}$}

In a final step, estimates for the parameters in $\Psi^{*}$ can be calculated, as suggested before, by taking averages of all the available estimates for that specific parameter. Obviously, this implies that $\hat{\Psi}^{*}=A^{\prime} \widehat{\Psi}$ for an appropriate weight matrix $A$. Hence, inference for the elements in $\hat{\Psi}^{*}$ will be based on

$$
\sqrt{N}\left(\widehat{\Psi}^{*}-\Psi^{*}\right)=\sqrt{N}\left(A^{\prime} \widehat{\Psi}-A^{\prime} \Psi\right) \approx N\left(0, A^{\prime} I_{0}^{-1} I_{1} I_{0}^{-1} A\right)
$$

As mentioned in Molenberghs and Verbeke ${ }^{[25]}$, pseudolikelihood methods are often less efficient than full maximum likelihood. However, simulation results of Fieuws et al. ${ }^{[46]}$, Fieuws and Verbeke ${ }^{[54]}$ suggest that, in the present context, this loss of efficiency is negligible, if any.

Wiley StatsRef: Statistics Reference Online, ( ) 2014-2018 John Wiley \& Sons, Ltd. 
To illustrate the pairwise approach for fitting high-dimensional multivariate repeated measurements, Fieuws et al. ${ }^{[46]}$ analyzed data from an experiment in which 105 Dutch-speaking elderly participants (54 females and 51 males) were randomly assigned to one of two physical activity-oriented exercise programs. The first is a classical fitness program consisting of three weekly visits to the gym. The second is a distance coaching program with an emphasis on incorporating physical activities in daily life. One of the aims of the study was to investigate whether the two programs have different impacts on the psychocognitive functioning of the participants. Different aspects of psychocognitive functioning referring to subjective well-being, self-esteem, self-perception, and motivation were considered. A set of questionnaires has been used to measure these different aspects. More specifically, seven sets of questions (items) were used, originating from different questionnaires and each set consisting of a different number of items: 10 items measuring physical well-being, 14 items for psychological well-being, 10 items for self-esteem, 30 items for physical self-perception, 21 items measuring the degree of opposition to physical activities, 5 items for perceived self-efficacy toward physical activity, and 16 items for motivation for the intervention program. All item scores were dichotomized, with a score equal to one expressing positive psychocognitive functioning. All subjects filled in at least one item for each of the seven sets. Sixty-four subjects had no missing information for the 106 items. Twenty subjects had one item missing. The missing item scores for the other subjects ranged from 2 to 22 . The mean age equals 66.6 years (range $60-76$ years) and the mean body mass index (BMI) is $27.0 \mathrm{kgm}^{-2}$ (range 20.7-38.0). Questionnaires considered in this analysis were completed by the participants, 6 months after the start of the study.

The aim of our analyses is to assess differences in efficacy between both exercise programs, as well as to study the strength of association between the seven sets of questionnaires. Although not of a longitudinal nature, this data set is clearly an example of multivariate repeated measurements, of dimension 7 , where a number of binary repeated measurements of psychocognitive functioning are available for each dimension. The random variable $Y_{r i j}$ now denotes the $j$ th measurement $(0$ or 1$)$, taken on the $i$ th study participant, for the $r$ th questionnaire, $i=1, \ldots, 105, r=1, \ldots, 7$, and $j=1, \ldots, n_{r i}$. A score $Y_{r i j}=1$ reflects positive psychocognitive functioning, while $Y_{r i j}=0$ is an indication of negative psychocognitive functioning.

We will assume that each of the seven questionnaires satisfy a random-intercepts logistic model, given by

$$
\operatorname{logit}\left[P\left(Y_{r i j}=1\right)\right]=\beta_{r 0}+\beta_{r 1} \mathrm{DC}_{i}+b_{r i}
$$

in which $\mathrm{DC}_{i}$ is an indicator variable equal to 1 for the participants in the distance coaching program, and zero otherwise. Hence, $\exp \left(\beta_{r 1}\right)$ represents the multiplicative effect of this program on the odds for positive psychocognitive functioning measured by the items in questionnaire $r$, with $r=1, \ldots, 7$ ( $1=$ physical wellbeing, $2=$ psychological well-being, $3=$ self-esteem, $4=$ physical self-perception, $5=$ degree of opposition, $6=$ self-efficacy, and $7=$ motivation). Note that this model allows for questionnaire-specific intercepts as well as intervention effects. More parsimonious models could be obtained by assuming, for example, the same regression parameters for all questionnaires, or by assuming some random effects to be common to a subset of the questionnaires (i.e., some of the $b_{r i}$ are equal). A correlation between the items of the same set is modeled through the inclusion of the random effects $b_{r i}$. A correlation between the items of the different questionnaires is implied by the joint distribution for the seven random intercepts, that is,

$$
\left(b_{1 i}, b_{2 i}, b_{3 i}, b_{4 i}, b_{5 i}, b_{6 i}, b_{7 i}\right)^{\prime} \sim N(0, \boldsymbol{D}),
$$

where $D$ is now the $7 \times 7$ unstructured covariance matrix of the random intercepts.

Table 9 shows the results from fitting the seven univariate models separately, as well as from fitting the joint model using the pairwise fitting approach. Very similar estimates as well as inferences are obtained. Using approximate Wald-type tests ( $Z$-tests), the separate analyses show significant differences between 


\section{Mixed Outcomes}

Table 9. Psychocognitive Functioning. Parameter estimates (standard errors) for the fixed effects in Model (31) obtained by fitting seven separate univariate models, as well as obtained by fitting the joint model with the pairwise fitting approach.

\begin{tabular}{lcr}
\hline & \multicolumn{2}{c}{ Seven univariate models } \\
\cline { 2 - 3 } & $\hat{\beta}_{r 0}$ (s.e.) & \multicolumn{1}{c}{$\hat{\beta}_{r 1}$ (s.e.) } \\
\hline Physical well-being & $1.63(0.26)$ & $-0.13(0.37)$ \\
Psychological well-being & $1.56(0.30)$ & $1.22(0.61)$ \\
Self-esteem & $1.69(0.30)$ & $0.43(0.42)$ \\
Physical self-perception & $-0.55(0.14)$ & $0.58(0.24)$ \\
Degree of opposition & $1.48(0.17)$ & $0.06(0.24)$ \\
Self-efficacy & $1.71(0.25)$ & $-0.24(0.33)$ \\
Motivation & $0.95(0.11)$ & $-0.35(0.16)$ \\
& \multicolumn{2}{c}{ Joint model } \\
\cline { 2 - 3 } Physical well-being & $1.62(0.25)$ & $-0.12(0.37)$ \\
Psychological well-being & $1.71(0.32)$ & $1.00(0.68)$ \\
Self-esteem & $1.68(0.32)$ & $0.49(0.39)$ \\
Physical self-perception & $-0.52(0.14)$ & $0.52(0.25)$ \\
Degree of opposition & $1.47(0.17)$ & $0.07(0.24)$ \\
Self-efficacy & $1.70(0.23)$ & $-0.22(0.33)$ \\
Motivation & $0.94(0.09)$ & $-0.34(0.16)$ \\
\hline
\end{tabular}

both groups on three of the seven questionnaires. The DC-group scores better on physical self-perception and on psychological well-being, but worse on motivation.

Using the results from the joint model, an overall test can be constructed for the presence of any systematic difference between both exercise programs. Formally, this corresponds to testing the null hypothesis

$$
H_{0}: \beta_{11}=\beta_{21}=\beta_{31}=\beta_{41}=\beta_{51}=\beta_{61}=\beta_{71}=0
$$

versus the alternative that at least one of these parameters differs from zero. As this null hypothesis is of the general form $H_{0}: L^{\prime} \Psi^{*}=0$ for the appropriate matrix $L$, a Wald-type test ( $\chi^{2}$-test) can easily be derived from the asymptotic distribution (Equation 30) for $\hat{\Psi}^{*}$. This yields a test statistic value equal to 17.84, which is significant when compared to the $\chi_{7}^{2}$ distribution $(p=0.013)$. Similarly, other hypotheses of interest can be tested as well.

An additional aim of our analyses was to study the strength of association between the seven sets of questionnaires. Table 10 presents the correlations obtained from the fitted covariance matrix $\widehat{D}$. These correlations express the association between the different constructs underlying each of the seven scales. Performing a principal components analysis (PCA) on the $7 \times 7$ correlation matrix of the random effects reveals that the first principal component explains only $49 \%$ of the variability. One approach sometimes used to join multiple random-effects models in such a way that the joint model can still easily be fitted using standard software, assumes common random effects for all outcomes, leading to so-called sharedparameter models. An example in a slightly different context can be found in DeGruttola and $\mathrm{Tu}^{[55]}$. More specifically, it is then assumed that all $b_{r i}$ equal $b_{i}$. In our example, this would lead to univariate random intercepts common to all questionnaires. The advantage would be that this model can very easily be fitted because only one random effect is involved. However, the PCA results suggest that this would be a very unrealistic model for the data set at hand, which could result in biased inferences for the fixed effects of interest ${ }^{[56,57]}$.

Wiley StatsRef: Statistics Reference Online, @ 2014-2018 John Wiley \& Sons, Ltd. 
Table 10. Psycho-cognitive functioning. Estimated correlation matrix for the random intercepts in Model 31.

\begin{tabular}{llllllll}
\hline Physical well-being & 1.00 & & & & & & \\
Psychological well-being & 0.75 & 1.00 & & & & & \\
Self-esteem & 0.55 & 0.76 & 1.00 & & & & \\
Physical self-perception & 0.66 & 0.46 & 0.53 & 1.00 & & \\
Degree of opposition & 0.19 & 0.12 & 0.23 & 0.38 & 1.00 & \\
Self-efficacy & 0.29 & 0.24 & 0.25 & 0.36 & 0.23 & 1.00 & \\
Motivation & 0.42 & 0.31 & 0.28 & 0.40 & 0.47 & 0.30 & 1.00 \\
\hline
\end{tabular}

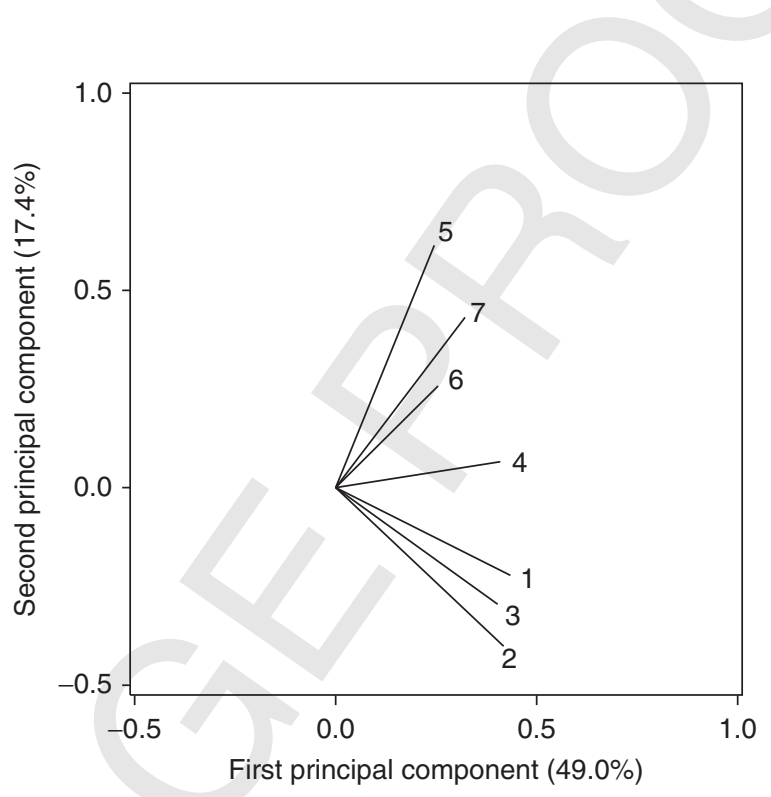

Figure 1. Psycho-cognitive functioning. Component loadings for the seven questionnaires on the first two principal components for the $7 \times 7$ correlation matrix of the random intercepts in Model (31). (1) physical well-being; (2) psychological well-being; (3) self-esteem; (4) physical self-perception; (5) degree of opposition; (6) self-efficacy; (7) motivation.

Figure 1 plots the component loadings of the random intercepts for the seven questionnaires on the first two principal components, explaining $49 \%$ and $17.4 \%$ of the variation. In this reduced representation, we observe, not surprisingly, that the scales referring to well-being and self-esteem are strongly correlated with each other, as opposed to their relation with motivational oriented scales.

\section{Software Considerations}

Methods based on shared and/or correlated random effects can easily be implemented in most mixed models software tools that allow for different data types and/or nonlinear model formulations. For example, the SAS procedures GLIMMIX and NLMIXED allow for multivariate longitudinal data of different data types. Note that, in particular, the longitudinal sequences can be of length one, which would bring us back to the simple mixed outcome cases. 
In the GLMM framework, it is recommended to use numerical integration over the random effects (available in the SAS procedures GLIMMIX and NLMIXED), rather than PQL, MQL, or Laplace approximations.

Note that the SAS procedure GLIMMIX is also useful for purely marginal semiparametric models that can be seen as a version of GEE as well.

Some factorization-based models are relatively easy to fit because they split into, for example, a linear regression and a logistic regression model, or into their longitudinal counterparts. For the model discussed in Section 6, Fieuws et al. ${ }^{[46]}$, Fieuws and Verbeke ${ }^{[54]}$ have developed a SAS macro, available at www.ibiostat.be.

Overall, thanks to recent software developments, the joint modeling of repeated measures of various outcome types can be done with standard statistical software and is not confined any more to user-defined program.

\section{Concluding Remarks}

We have discussed a number of methods to model correlated data when not all outcomes are of the same type. It is not uncommon to observe binary or otherwise categorical outcomes jointly with continuous outcomes, but also other combinations are perfectly well possible. One might view such outcomes as multivariate. In addition, such a multivariate outcome of a heterogeneous nature can then be observed repeatedly over time, for various subjects within a trial, a cluster, or within other hierarchically organized units. Just as in the general case, we have distinguished between marginal, conditional, and random-effects models. A relatively large number of proposals have been made in the literature, many developed for specific applications. Specific attention was given to applications in surrogate marker evaluation on the one hand, and risk assessment in the context of developmental toxicity studies on the other.

In principle, the joint modeling of longitudinal and time-to-event data could also be considered a form of mixed outcome. However, that particular case is typically treated under the denominator of joint models, an excellent account of which is given in ${ }^{[58]}$.

\section{Acknowledgments}

Financial support from the IAP research network \#P7/06 of the Belgian Government (Belgian Science Policy) is gratefully acknowledged. The research leading to these results has also received funding from the European Seventh Framework programme FP7 2007-2013 under grant agreement No 602552.

\section{Related Articles}

Dose-Response Modeling for Clustered Data; Correlated Multinomial Data; Reproductive Toxicology; Environmental Health Risk Assessment, Statistics in; Dose-Response Models in Risk Analysis; Competing Risk Analysis; Generalized Linear Models for Longitudinal Data; Repeated Measurements; Mixed Effects; Linear Mixed Effects Models for Longitudinal Data; Joint Modelling of Longitudinal and Event Time Data; Generalized Linear Models for Longitudinal Data; Joint Models; Joint Modeling of Longitudinal and Survival Data; Composite Likelihood/Pseudolikelihood; Pseudo-Likelihood; Pseudo-Likelihood Function.

Wiley StatsRef: Statistics Reference Online, ( $\odot$ 2014-2018 John Wiley \& Sons, Ltd. 


\section{References}

[1] Tate, R.F. (1954) Correlation between a discrete and a continuous variable. Ann. Math. Stat. 25, 603-607.

[2] Cox, D.R. and Wermuth, N. (1992) Response models for mixed binary and quantitative variables. Biometrika 79, 441 -461.

[3] Cox, D.R. and Wermuth, N. (1994) Multivariate Dependencies: Models, Analysis and Interpretation, Chapman and Hall, London.

[4] Krzanowski, W.J. (1988) Principles of Multivariate Analysis: A User's Perspective, Clarendon Press, Oxford.

[5] Little, R.J.A. and Schluchter, M.D. (1985) Maximum likelihood estimation for mixed continuous and categorical data with missing values. Biometrika 72, 497-512.

[6] Schafer, J.L. (1997) Analysis of Incomplete Multivariate Data, Chapman and Hall, London.

[7] Tate, R.F. (1955) The theory of correlation between two continuous variables when one is dichotomized. Biometrika 42 205-216.

[8] Molenberghs, G., Geys, H., and Buyse, M. (2001) Validation of surrogate endpoints in randomized experiments with mixed discrete and continuous outcomes. Stat. Med. 20, 3023-3038.

[9] Prentice, R.L. and Zhao, L.P. (1991) Estimating equations for parameters in means and covariances of multivariate discrete and continuous responses. Biometrics 47, 825-839.

[10] Zhao, L.P., Prentice, R.L., and Self, S.G. (1992) Multivariate mean parameter estimation by using a partly exponential model. J. R. Stat. Soc., Ser. B 54, 805-811.

[11] Sammel, M.D., Ryan, L.M., and Legler, J.M. (1997) Latent variable models for mixed discrete and continuous outcomes. J. R. Stat. Soc., Ser. B, 59, 667-678.

[12] Olkin, I. and Tate, R.F. (1961) Multivariate correlation models with mixed discrete and continuous variables. Ann. Math. Stat. 32, 448-465 (with correction in 36, 343-344).

[13] Hannan, J.F. and Tate, R.F. (1965) Estimation of the parameters for a multivariate normal distribution when one variable is dichotomized. Biometrika 52, 664-668.

[14] Cox, N.R. (1974) Estimation of the correlation between a continuous and a discrete variable. Biometrics 30, 171-178.

[15] Regan, M.M., Catalano, P.J., Aerts, M., et al. (2002) Combined continuous and discrete outcomes, in Topics in Modelling of Clustered Data, Chapman \& Hall, London.

[16] Catalano, P.J. and Ryan, L.M. (1992) Bivariate latent variable models for clustered discrete and continuous outcomes. J. Am. Stat. Assoc. 87, 651-658.

[17] Fitzmaurice, G.M. and Laird, N.M. (1995) Regression models for a bivariate discrete and continuous outcome with clustering. J. Am. Stat. Assoc. 90, 845-852.

[18] Catalano, P.J. (1997) Bivariate modelling of clustered continuous and ordered categorical outcomes. Stat. Med. 16, $883-900$.

[19] Regan, M.M. and Catalano, P.J. (1999) Likelihood models for clustered binary and continuous outcomes: application to developmental toxicology. Biometrics 55, 760-768.

[20] Ochi, Y. and Prentice, R.L. (1984) Likelihood inference in a correlated probit regression model. Biometrika 71, 531 -543.

[21] Geys, H., Regan, M., Catalano, P., and Molenberghs, G. (2001) Two latent variable risk assessment approaches for combined continuous and discrete outcomes from developmental toxicity data. J. Agric. Biol. Environ. Stat. 6, $340-355$.

[22] Liang, K.Y. and Zeger, S. (1986) Longitudinal data analysis using generalized linear models. Biometrika 73, 13-22.

[23] Regan, M.M. and Catalano, P.J. (1999) Bivariate dose-response modeling and risk estimation in developmental toxicology. J. Agric. Biol. Environ. Stat. 4, 217-237.

[24] Regan, M.M. and Catalano, P.J. (2000) Regression models for mixed discrete and continuous outcomes with clustering. Risk Anal. 20, 363-376.

[25] Molenberghs, G. and Verbeke, G. (2005) Models for Discrete Longitudinal Data, Springer-Verlag, New York.

[26] Burzykowski, T., Molenberghs, G., and Buyse, M. (2005) The Evaluation of Surrogate Endpoints, Springer-Verlag, New York.

[27] Alonso, A., Bigirumurame, T., Burzykowski, T., et al. (2017) Applied Surrogate Endpoint Evaluation with SAS and R, Chapman \& Hall/CRC, Boca Raton, FL.

[28] Plackett, R.L. (1965) A class of bivariate distributions. J. Am. Stat. Assoc. 60, 516-522

[29] Wolfinger, R. and O'Connell, M. (1993) Generalized linear mixed models: a pseudo-likelihood approach. J. Stat. Comput. Simul. 48, 233-243.

[30] Breslow, N.E. and Clayton, D.G. (1993) Approximate inference in generalized linear mixed models. J. Am. Stat. Assoc. 88, 9-25.

[31] Pharmacological Therapy for Macular Degeneration Study Group (1997) Interferon $\alpha$-IIA is ineffective for patients with choroidal neovascularization secondary to age-related macular degeneration. Results of a prospective randomized placebo-controlled clinical trial. Arch. Ophthalmol. 115, 865-872. 
[32] Buyse, M. and Molenberghs, G. (1998) The validation of surrogate endpoints in randomized experiments. Biometrics 54, 1014-1029.

[33] Buyse, M., Molenberghs, G., Burzykowski, T., et al. (2000) The validation of surrogate endpoints in meta-analyses of randomized experiments. Biostatistics 1, 49-67.

[34] Aerts, M., Geys, H., Molenberghs, G., and Ryan, L. (2002) Topics in Modelling of Clustered Data, Chapman \& Hall/CRC, London.

[35] U.S. Environmental Protection Agency (1991) Guidelines for developmental toxicity risk assessment. Fed. Regist. 56, $63798-63826$

[36] Chen, J.J. and Kodell, R.L. (1989) Quantitative risk assessment for teratologic effects. J. Am. Stat. Assoc. 84, 966-971.

[37] Kupper, L.L., Portier, C., Hogan, M.D., and Yamamoto, E. (1986) The impact of litter effects on dose-response modeling in teratology. Biometrics 42, 85-98.

[38] Chen, J.J. and Gaylor, D.W. (1992) Correlations of developmental end points observed after 2,4,5-trichlorophenoxyacetic acid exposure in mice. Teratology 45, 241-246.

[39] Ryan, L.M., Catalano, P.J., Kimmel, C.A., and Kimmel, G.L. (1991) Relationship between fetal weight and malformation in developmental toxicity studies. Teratology 44, 215-223.

[40] Fitzmaurice, G.M. and Laird, N.M. (1997) Regression models for mixed discrete and continuous responses with potentially missing values. Biometrics 53, 110-122.

[41] Ahn, H. and Chen, J.J. (1997) Tree-structured logistic models for over-dispersed binomial data with application to modeling developmental effects. Biometrics 53, 435-455.

[42] Chen, J. (1993) A malformation incidence dose-response model incorporating fetal weight and/or litter size as covariates. Risk Anal. 13, 559-564.

[43] Catalano, P.J., Scharfstein, D.O., Ryan, L.M., et al. (1993) Statistical model for fetal death, fetal weight, and malformation in developmental toxicity studies. Teratology 47, 281-290.

[44] Geys, H., Molenberghs, G., and Ryan, L. (1999) Pseudo-likelihood modelling of multivariate outcomes in developmental toxicology. J. Am. Stat. Soc. 94, 734-745.

[45] Fieuws, S. and Verbeke, G. (2004) Joint modelling of multivariate longitudinal profiles: pitfalls of the random-effects approach. Stat. Med. 23, 3093-3104

[46] Fieuws, S. and Verbeke, G. (2006) Pairwise fitting of mixed models for the joint modelling of multivariate longitudinal profiles. Biometrics 62, 424-431.

[47] Burzykowski, T., Molenberghs, G., Buyse, M., et al. (2001) Validation of surrogate endpoints in multiple randomized clinical trials with failure time end points. Appl. Stat. 50, 405-422.

[48] Thum, Y.M. (1997) Hierarchical linear models for multivariate outcomes. J. Educ. Behav. Stat. 22, 77-108.

[49] Gueorguieva, R. (2001) A multivariate generalized linear mixed model for joint modelling of clustered outcomes in the exponential family. Stat. Model. 1, 177-193.

[50] Chakraborty, H., Helms, R.W., Sen, P.K., and Cohen, M.S. (2003) Estimating correlation by using a general linear mixed model: evaluation of the relationship between the concentration of HIV-1 RNA in blood and semen. Stat. Med. 22, $1457-1464$.

[51] MacCallum, R., Kim, C., Malarkey, W., and Kiecolt-Glaser, J. (1997) Studying multivariate change using multilevel models and latent curve models. Multivariate Behav. Res. 32, 215-253.

[52] Thiébaut, R., Jacqmin-Gadda, H., Chêne, G., et al. (2002) Bivariate linear mixed models using SAS PROC MIXED. Comput. Methods Programs Biomed. 69, 249-256.

[53] Shah, A., Laird, N., and Schoenfeld, D. (1997) A random-effects model for multiple characteristics with possibly missing data. J. Am. Stat. Assoc. 92, 775-779,

[54] Fieuws, S., Verbeke, G., Boen, F., and Delecluse, C. (2006) High-dimensional multivariate mixed models for binary questionnaire data. Appl. Stat. 55, 1-12.

[55] DeGruttola, V. and Tu, X. (1994) Modeling progression of CD-4 lymphocyte count and its relationship to survival time. Biometrics 50, 1003-1014.

[56] Adams, R.J., Wilson, M., and Wang, W. (1997) The multidimensional random coefficients multinomial logit model. Appl. Psychol. Meas. 21, 1-23.

[57] Folk, V.G. and Green, B.F. (1989) Adaptive estimation when the unidimensionality assumption of irt is violated. Appl. Psychol. Meas. 13, 373-389.

[58] Rizopoulos, D. (2012) Joint Models for Longitudinal and Time-to-Event Data. With Applications in R, Chapman \& Hall/CRC, Boca Raton, FL. 